ARTICLE

\title{
Structural basis for voltage-sensor trapping of the cardiac sodium channel by a deathstalker scorpion toxin
}

Daohua Jiang ${ }^{1}$, Lige Tonggu (10 1, Tamer M. Gamal El-Din¹, Richard Banh2,3, Régis Pomès (D) 2,3, Ning Zheng $\mathbb{B}^{1,4 \times} \&$ William A. Catterall (i) ${ }^{1 凶}$

Voltage-gated sodium ( $\mathrm{Nav}_{\mathrm{v}}$ ) channels initiate action potentials in excitable cells, and their function is altered by potent gating-modifier toxins. The $\alpha$-toxin LqhIll from the deathstalker scorpion inhibits fast inactivation of cardiac Nav1.5 channels with $I C_{50}=11.4 \mathrm{nM}$. Here we reveal the structure of LqhIII bound to $\mathrm{Na}_{V} 1.5$ at $3.3 \AA$ resolution by cryo-EM. LqhIII anchors on top of voltage-sensing domain IV, wedged between the S1-S2 and S3-S4 linkers, which traps the gating charges of the $\mathrm{S} 4$ segment in a unique intermediate-activated state stabilized by four ion-pairs. This conformational change is propagated inward to weaken binding of the fast inactivation gate and favor opening the activation gate. However, these changes do not permit $\mathrm{Na}^{+}$permeation, revealing why LqhIll slows inactivation of $\mathrm{Na}_{V}$ channels but does not open them. Our results provide important insights into the structural basis for gating-modifier toxin binding, voltage-sensor trapping, and fast inactivation of $\mathrm{Na}_{\vee}$ channels.

\footnotetext{
${ }^{1}$ Department of Pharmacology, University of Washington, Seattle, WA 98195, USA. ${ }^{2}$ Molecular Medicine, Hospital for Sick Children, Toronto, ON M5G OA4, Canada. ${ }^{3}$ Department of Biochemistry, University of Toronto, Toronto, ON M5S1A8, Canada. ${ }^{4}$ Howard Hughes Medical Institute, University of Washington, Seattle, WA 98195, USA.『email: nzheng@uw.edu; wcatt@uw.edu
} 
E ukaryotic voltage-gated sodium $\left(\mathrm{Na}_{\mathrm{V}}\right)$ channels generate the inward sodium current that is responsible for initiating and propagating action potentials in nerve and muscle ${ }^{1,2}$. The sodium current is terminated within $1-2 \mathrm{~ms}$ by fast inactivation $^{1,2}$. A wide variety of neurotoxins bind to six distinct receptor sites on $\mathrm{Na}_{\mathrm{V}}$ channels and modify their function ${ }^{3,4}$. $\alpha$-Scorpion toxins and sea anemone toxins bind to Neurotoxin Receptor Site 3 , dramatically inhibit fast inactivation of $\mathrm{Na}_{\mathrm{V}}$ channels, and cause prolonged and/or repetitive action potentials ${ }^{3-5}$. Scorpions utilize these toxins in their venoms to immobilize prey by inducing paralysis and causing cardiac arrhythmia ${ }^{4,6-8}$. Because of their high affinity and specificity, scorpion toxins are used widely to study the structure and function of $\mathrm{Na}_{\mathrm{V}}$ channels. $\alpha$-Scorpion toxins bind to the voltage sensor (VS) in domain IV (DIV), which is important for triggering fast inactivation ${ }^{9-13}$. Therefore, structures of the high-affinity complexes of $\alpha$-scorpion toxins and $\mathrm{Na}_{V}$ channels will provide critical information for understanding the structural basis for toxin binding, voltage-sensor trapping, and fast inactivation.

Eukaryotic $\mathrm{Na}_{\mathrm{V}}$ channels contain four homologous, nonidentical domains composed of six transmembrane segments (S1-S6), organized into a voltage-sensing module (VS, S1-S4) and a pore module (PM, S5-S6) with two intervening pore helices (P1 and P2) ${ }^{14,15}$. The S4 segments contain four to eight repeats of a positively charged residue (usually Arg) flanked by two hydrophobic residues. These positively charged residues serve as gating charges, moving outward upon depolarization to initiate the process of activation ${ }^{14,15}$. Chemical labeling and voltage clamp fluorometry suggest that DI-VS and DII-VS are primarily responsible for activation of the channel, whereas DIV-VS induces fast inactivation ${ }^{14,15}$. A triple hydrophobic motif, Ile-Phe-Met (IFM), located in the DIII-DIV linker, serves as the fast inactivation gate ${ }^{14,15}$. Mutation of the IFM motif can completely abolish fast inactivation ${ }^{14,15}$.

Determination of the structures of prokaryotic ${ }^{16-18}$ and eukaryotic $^{19-21} \mathrm{Na}_{\mathrm{V}}$ channels has remarkably enriched our understanding of their structure and function. Those structures revealed that $\mathrm{Na}_{\mathrm{V}}$ channels share similar key structural features ${ }^{22}$. The central pore is formed by the four PMs with the four VSs arranged in a pseudosymmetric square array on their periphery. The four homologous domains are organized in a domainswapped manner, in which each VS interacts most closely with the PM of the neighboring domain. The four S6 segments come together at their intracellular ends to form the activation gate $e^{16-18}$. Intriguingly, in the structures of mammalian $\mathrm{Na}_{\mathrm{V}} \mathrm{s}$, the IFM motif binds in a receptor site formed by the DIII S4-S5 linker and the intracellular ends of the S5 and S6 segments in DIV, which suggests a local allosteric mechanism for fast inactivation of the pore by closing the intracellular activation gate ${ }^{19-21}$.

The a-scorpion toxins bind to DIV-VS in its resting state, trap it in an intermediate-activated conformation, and inhibit fast inactivation, providing an attractive target for studying the coupling of DIV-VS to pore opening and fast inactivation ${ }^{9-13,23,24}$. Strong depolarization can reverse voltage-sensor trapping and drive the a-scorpion toxin off its receptor site, providing direct evidence for a toxin-induced conformation of the $\mathrm{VS}^{9,24,25}$. The cryo-EM structure of the a-scorpion toxin AaHII was resolved bound to two different sites on a nonfunctional chimera of the cockroach sodium channel $\mathrm{Na}_{\mathrm{V}} \mathrm{Pas}$, which contained 132 amino acid residues of the DIV-VS of the human neuronal sodium channel $\mathrm{Na}_{\mathrm{V}} 1.7$ embedded within 1449 residues of $\mathrm{Na}_{\mathrm{V}} \mathrm{Pas}^{26}$. These results revealed structures of AaHII bound to the VSs in both DI and DIV but did not resolve whether AaHII bound to either of these sites was functionally active in the chimera ${ }^{26}$. Therefore, the precise structural mechanism by which $\alpha$-scorpion toxin binds to the DIV-VS in a native sodium channel and blocks fast inactivation still remains elusive.
LqhIII from the "deathstalker scorpion" Leiurus quinquestriatus hebraeus (also known as the Israeli yellow scorpion and the North African striped scorpion) is classified as an $\alpha$-scorpion toxin and shares the common $\beta a \beta \beta$ scaffold containing four pairs of Cys residues that form disulfide bonds ${ }^{7}$. Most scorpion toxins paralyze prey by targeting the sodium channels in nerve and skeletal muscle specifically ${ }^{7}$. In contrast, LqhIII binds with the highest affinity to the human cardiac sodium channel, with an estimated $\mathrm{EC}_{50}$ of $2.5 \mathrm{nM}^{27,28}$. It prevents fast inactivation efficiently, and it dissociates at an extremely slow rate ${ }^{27,28}$, making it exceptionally potent.

In this work, we elucidate the molecular mechanisms of voltage-sensor trapping and block of fast inactivation by a-scorpion toxins in the context of a functional native toxin-receptor complex by determining the cryo-EM structure of rat cardiac sodium channel $\mathrm{Na}_{\mathrm{V}} 1.5$ in complex with the $\alpha$-scorpion toxin LqhIII at $3.3 \AA$ resolution. Our experiments provide important insights into the structural basis for gating-modifier toxin interaction, voltage-sensor trapping, electromechanical coupling in the VS, and fast inactivation of the pore.

\section{Results}

Voltage-sensor trapping of $\mathrm{Na}_{\mathrm{V}} 1.5$ by LqhIII. For our structural studies, we took advantage of the fully functional core construct of the rat cardiac sodium channel $\mathrm{Na}_{\mathrm{V}} 1.5\left(\mathrm{rNa}_{\mathrm{V}} 1.5_{\mathrm{C}}\right)$, which can be isolated with high yield and high stability ${ }^{21}$. Expression of $\mathrm{rNa}_{\mathrm{V}} 1.5_{\mathrm{C}}$ in the human embryonic kidney cell line HEK293S $\mathrm{GnTI}^{-}$and recording from single cells in whole-cell patch clamp mode (see "Methods") yields inward sodium currents that activate rapidly and inactivate within $6 \mathrm{~ms}$ (Fig. 1a, black trace; the inward current is plotted as a negative quantity by convention). Perfusion of increasing concentrations of LqhIII progressively slows the fast inactivation process and makes it incomplete (Fig. 1a, colored traces). We measured the sodium current remaining $6 \mathrm{~ms}$ after the depolarizing pulse as a metric of LqhIII toxin action (Fig. 1a, dotted line), because the unmodified sodium current has declined to nearly zero by this time, whereas substantial toxin-modified sodium current remains. The $\mathrm{EC}_{50}$ value for the increase in sodium current remaining at $6 \mathrm{~ms}$ following the stimulus is $11.4 \mathrm{nM}$ (Fig. 1a). This effect of LqhIII and other a-scorpion toxins is achieved by trapping the VS in DIV of sodium channels in a conformation that allows sodium channel activation but prevents coupling to fast inactivation ${ }^{4,9,10}$. Voltagesensor trapping develops slowly and progressively over more than $20 \mathrm{~min}$, with a half-time of $11.3 \mathrm{~min}$ at $100 \mathrm{nM}$ (Fig. 1b). As expected from previous work $4,9,10$, strong depolarizing pulses to $+100 \mathrm{mV}$ cause dissociation of the toxin and loss of its blocking effect on fast inactivation (Fig. 1c). The molecular mechanism for this long-lasting voltage-dependent block of fast inactivation of $\mathrm{Na}_{\mathrm{V}} 1.5$ sodium currents by LqhIII is unknown.

Structure determination of $\mathrm{rNa}_{\mathrm{V}} 1.5_{\mathrm{C}}$ /LqhIII complex by cryoEM. We analyzed the structural basis for the potent voltagesensor trapping effects of LqhIII by cryogenic electron microscopy (cryo-EM). LqhIII was incubated with purified $\mathrm{rNa}_{\mathrm{V}} 1.5_{\mathrm{C}}$ for $30 \mathrm{~min}$. The regulatory proteins FGF12b and calmodulin were added to stabilize the isolated protein, and the toxin/channel complex was further purified by size-exclusion chromatography (SEC). A symmetric peak of the toxin/channel complex was collected from the second SEC run (Supplementary Fig. 1a, b). Detailed descriptions of protein expression, purification, cryo-EM imaging, and data processing are presented in "Methods".

Cryo-EM data were collected on a Titan-Krios electron microscope and processed using RELION (Supplementary Fig. 1c, d; Supplementary Fig. 2a-c). A 3D reconstruction map 
a

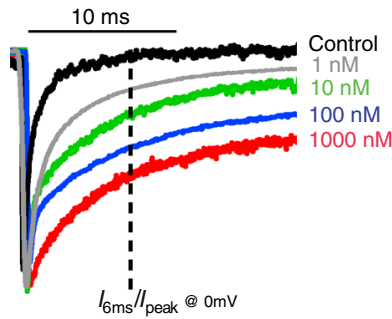

b

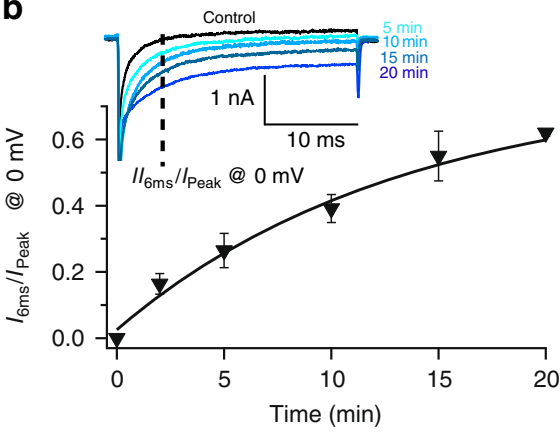

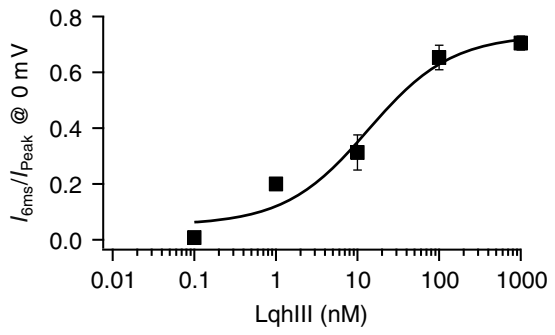

C

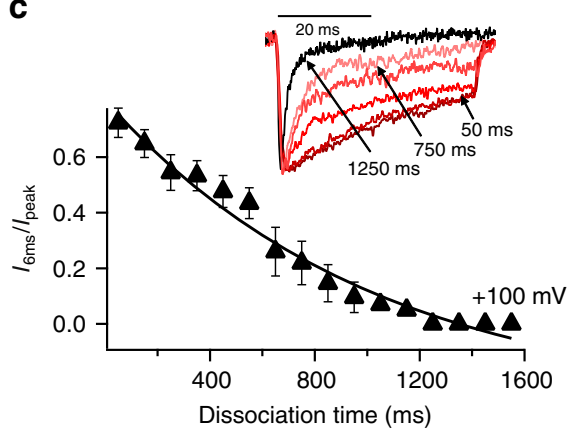

Fig. 1 Block of fast inactivation of $\mathbf{r N a} \mathbf{v} \mathbf{1 . 5} \mathbf{c}$ by LqhIII. a Left. Normalized current traces from HEK293 cells expressing rNa $\mathbf{r} 1.5 \mathrm{c}$ in the absence (black) or in the presence of $1 \mathrm{nM}$ (grey), $10 \mathrm{nM}$ (green), $100 \mathrm{nM}$ (blue), or $1000 \mathrm{nM}$ (red) Lqhlll. Cells were held at $-120 \mathrm{mV}$ and $\mathrm{Na}^{+}$currents were elicited with a 1000 -ms step to $0 \mathrm{mV}$. Measurements at different toxin concentrations were carried out on different cells because of the limited stability of the whole-cell recording configuration on virus-infected HEK293 cells. Right. Concentration-response curve of Lqhlll scorpion toxin for inhibition of fast inactivation of the $\mathrm{rNav} 1.5 \mathrm{c}$ channel. Each point is an average of $4-5$ cells. Data-points and error bars represent mean and s.e.m. The solid line represents the Hill equation fit to the data. $\mathrm{EC}_{50}=11.4 \pm 0.9 \mathrm{nM}$ (s.e.m., $n=23$ cells). $\mathbf{b}$ The time course of association of $100 \mathrm{nM}$ of Lqhlll scorpion toxin. Cells were held at $-120 \mathrm{mV}$ and the toxin was perfused. A pulse to $0 \mathrm{mV}$ from $V_{\mathrm{m}}=-120 \mathrm{mV}$ was applied at the indicated times. Single exponential fitting of the block of inhibition ratio showed a time constant of $11.3 \mathrm{~min}$. Each point is an average of six cells. Data points and error bars represent mean and s.e.m. c Time course of LqhIII dissociation. A three-pulse protocol was applied: first, a pulse from $-120 \mathrm{mV}$ to $+100 \mathrm{mV}$ for the indicated times, followed by a second $50-\mathrm{ms}$ hyperpolarizing pulse to allow recovery from fast inactivation, and finally by the third pulse of $50 \mathrm{~ms}$ to $0 \mathrm{mV}$ to measure the extent of recovery of fast inactivation kinetics. Mean and s.e.m.; $n=5$ cells for each data point. Inset, representative traces showing recovery of fast inactivation. Source Data are provided as a Source Data File.

of the $\mathrm{rNa}_{\mathrm{V}} 1.5_{\mathrm{C}}$ /LqhIII complex was obtained at an overall resolution of $3.3 \AA$, based on the Fourier Shell Correlation (FSC) between independently refined half-maps (Fig. 2a). Strong density specifically localized near the extracellular side of DIV-VS shows that there is only one LqhIII molecule bound to $\mathrm{rNa}_{\mathrm{V}} 1.5_{\mathrm{C}}$ (Fig. 2b; purple), as expected from previous biochemical studies of scorpion toxin binding to sodium channels ${ }^{9}$. The local resolution for the PM core region is $\sim 3.0-3.5 \AA$, whereas the four peripheral VSs have local resolutions of $\sim 3.5-4.0 \AA$ (Fig. $2 \mathrm{c}$ ). The resolution for the toxin is lower than the channel protein ( 4.0-5.0 $\AA$, Fig. 2c). However, the interacting surface of the toxin that binds to DIV-VS has a resolution of $\sim 3.5-4.0 \AA$ for the amino acid side chains that form the complex, as they are tightly bound (Supplementary Fig. 2d, e). The 3D structure of the tightly disulfide-crosslinked toxin is well-known from previous studies (Supplementary Fig. 3a) ${ }^{29}$, allowing it to be accurately fit into the observed density. No significant density was observed at high resolution for the C-terminal domain (CTD), FGF12b, or calmodulin (Fig. 2b), indicating that these components of the purified protein complex are mobile.

The overall structure and LqhIII binding site. The highresolution cryo-EM density map allowed us to build an atomic model for the $\mathrm{rNa}_{\mathrm{V}} 1.5_{\mathrm{C}}$ /LqhIII complex (Fig. 3a, b). The overall structure of the $\mathrm{rNa}_{\mathrm{V}} 1.5_{\mathrm{C}}$ /LqhIII complex is very similar to our previous apo$\mathrm{rNa}_{\mathrm{V}} 1.5_{\mathrm{C}}$ structure $^{21}$, with a minimum RMSD of $0.78 \AA$ over 1164 residues. However, local conformational differences give many important insights. The structure of LqhIII is rigidly locked by disulfide bonds, except for the $\beta 2 \beta 3$ loop and C-terminal region, which are highly flexible in solution as revealed by nuclear magnetic resonance analyses (Fig. 3c). Remarkably, LqhIII uses these two flexible regions to bind to the extracellular side of DIV-VS by wedging its $\beta 2 \beta 3$ loop and C-terminus into the aqueous cleft formed by the S1-S2 and S3-S4 helical hairpins (Fig. 3b). These features are in close agreement with previous molecular-mapping studies of neurotoxin receptor site $3^{11}$ and with the structure of the AaHII/ $\mathrm{Na}_{\mathrm{V}} \mathrm{Pas}-\mathrm{Na}_{\mathrm{V}} 1.7$ chimera $^{26}$ (see "Discussion"). The toxin may attack Neurotoxin Receptor Site 3 in the DIV-VS using its most flexible regions to allow it to dock in a stepwise manner that results in a tight induced-fit complex.

The close interactions of the C-terminus and the $\beta 2 \beta 3$ loop of LqhIII with $\mathrm{rNa}_{\mathrm{V}} 1.5_{\mathrm{C}}$ are illustrated in Fig. $3 \mathrm{~b}$, d. At the Cterminus of LqhIII, Glu63 interacts with the Asn329-linked glycan from DI-PM, and Lys64 dips into the aqueous cleft and interacts with Gln1615 (Fig. 3b, d). The end of the $\beta 2 \beta 3$ loop inserts into the DIV-VS cleft and partially unwinds the last helical turn of the S3 segment. Mutagenesis studies mapping Neurotoxin Receptor Site 3 revealed a negatively charged residue in the extracellular S3-S4 linker that is conserved among $\mathrm{Na}_{\mathrm{V}}$ channels and is critical for a-scorpion toxin binding ${ }^{4,9}$. In agreement with those studies, the conserved negatively charged residue Asp1612 at an equivalent position in $\mathrm{Na}_{\mathrm{V}} 1.5$ mediates this crucial interaction with the bound toxin. His43 and His15 wrap around Asp1612 like pincers forming a hydrogen bond $(\sim 2.5 \AA)$ and a potential salt bridge $(\sim 4.0 \AA)$, respectively (Fig. $3 \mathrm{~d})$. Moreover, we note that the backbone carbonyl of His43 engages the backbone carbonyl of Thr1608 at a distance of $2.8-3.5 \AA$, which may contribute to the affinity or specificity of interactions with the $\beta 2 \beta 3$ loop (Fig. 3d) ${ }^{30}$. 

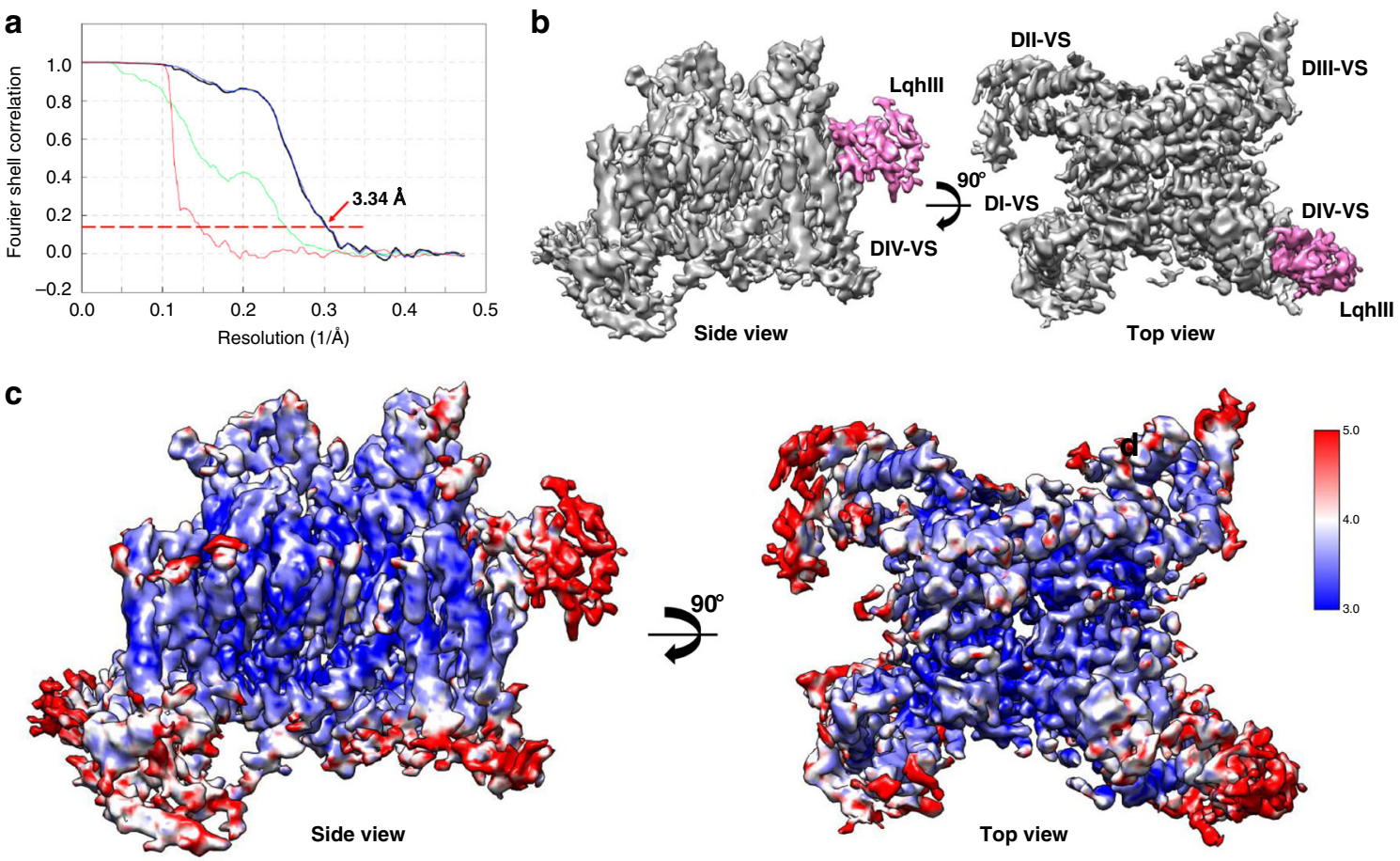

Fig. 2 Cryo-EM structure of the rNaV1.5c/LqhIII complex. a The FSC between independently refined half-maps for $r \mathrm{Na}_{\mathrm{v}} 1.5 \mathrm{C} / \mathrm{Lqh}$ III reconstruction. b Overall cryo-EM reconstruction (side view, left; top view, right) of the $\mathrm{rNa} v 1.5 \mathrm{C} /$ LqhIII complex. Nav $1.5_{\mathrm{C}}$ and LqhIII colored in grey and purple, respectively. c Local resolution (side view, left; top view, right) of the EM map colored from blue to red representing resolution from high to low (sidebar).

a

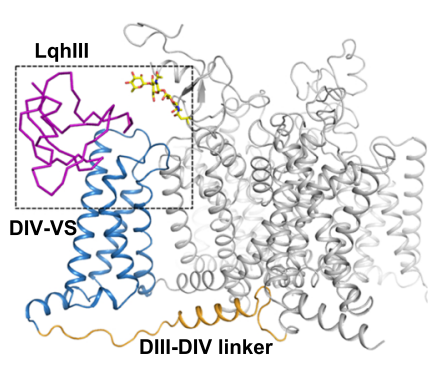

d

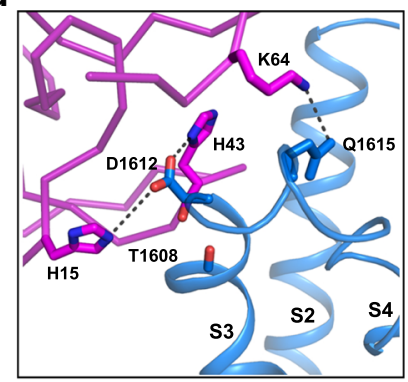

b

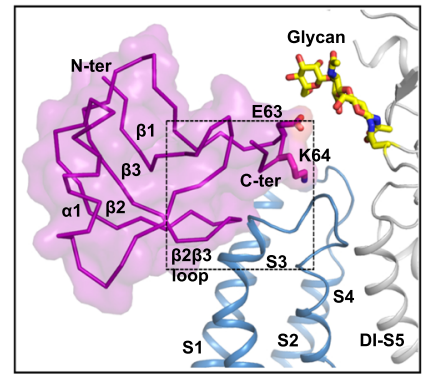

e

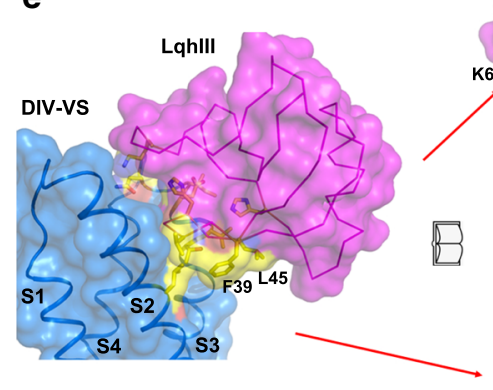

C
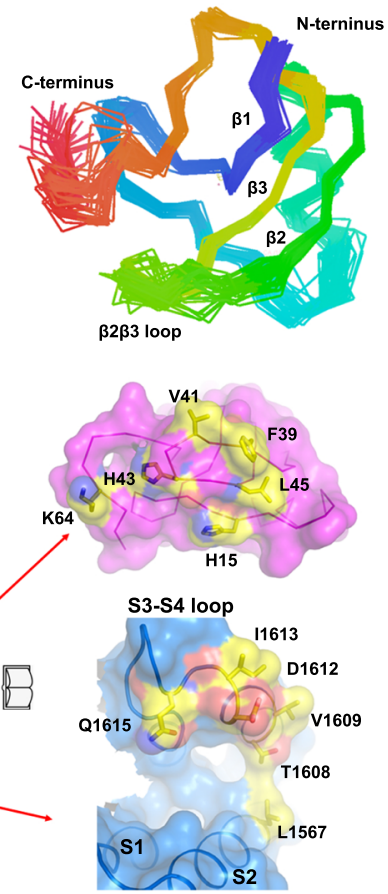

Fig. 3 Overall structure of $\mathbf{r N a v 1 . 5}$ /LqhIII complex and LqhIII binding site. a Cartoon representation of the overall structure of the Nav1.5c/LqhIII. LqhIII, DIV-VS and DIII-DIV linker were colored in purple, blue and orange, respectively. The same color scheme is applied hereafter in this paper unless specified otherwise. The glycosyl moieties are shown in sticks colored in yellow. The black dash square is indicated for panel (b). b Zoom-in view of Lqhlll binding to the DIV-VS. The black dash square indicated for panel (d). c The NMR structure of the LqhIII (PDB code: 1FH3) indicating the flexibility of the $\beta 2 \beta 3$ loop and C-terminus. d Detailed interactions between LqhIII and DIV-VS. Key residues shown in sticks were labeled. Interaction surfaces of the DIV-VS (blue) and the LqhIII (purple). Key residues for the interaction shown in yellow shading and embedded sticks. 
The complementary interacting surfaces of LqhIII and Neurotoxin Receptor Site 3 are depicted in a space-filling model in Fig. $3 \mathrm{e}$ (left), and the functionally important interacting residues are highlighted in yellow with embedded sticks and displayed in an "open-book" format in Fig. 3e (right). The interacting surface area of neurotoxin receptor site 3 covers $\sim 836 \AA^{2}$ located on an arc stretching from the S3-S4 linker to the S1-S2 linker (Fig. 3e, right). The LqhIII toxin latches onto that arc, gripping it between the $\beta 2 \beta 3$ loop and the C-terminus (Fig. 3e). It is likely that the flexibility of these regions of the toxin in solution is important for its initial approach and final tight grip on its target site.

An intermediate-activated-state of DIV-VS trapped by LqhIII. Fast inactivation of $\mathrm{Na}_{\mathrm{V}}$ channels requires activation of DIV$\mathrm{VS}^{9,10,12,13}$. Because there is no membrane potential during solubilization and purification, the VSs of published $\mathrm{Na}_{V}$ structures are usually in partially or fully activated states. In our apo$\mathrm{rNa}_{\mathrm{V}} 1.5_{\mathrm{C}}$ structure, four of the six gating charges of DIV-VS pointed outward on the extracellular side of the hydrophobic constriction site (HCS), as expected for an activated state ${ }^{21}$. As a result, the fast inactivation gate in the apo-rNa $1.5_{C}$ structure binds tightly in a hydrophobic pocket next to the activation gate $^{21}$. a-Scorpion toxins bind to $\mathrm{Na}_{\mathrm{V}}$ channels in the resting state with higher affinity and trap the channel in a partially activated state, in which both the rate and extent of transition to the inactivated state are impaired (Fig. 1) ${ }^{9,10}$. Because of its high affinity and specificity, LqhIII is able to bind to the purified $\mathrm{rNa}_{\mathrm{V}} 1.5_{\mathrm{C}}$ protein in its activated state and chemically induce voltage-dependent structural changes to partially deactivate the VS. Remarkably, LqhIII binding drives DIV-S4 approximately two helical turns inward to form an intermediate, partially activated structure (Fig. 4a, b). Each gating charge Arg in the intermediate-activated DIV-VS is positioned $\sim 10-12 \AA$ further inward than in the fully activated DIV-VS (Fig. 4a, b). Importantly, in the toxin-bound intermediate-activated state-reported here, R1-R4 adopts a 310 -helix conformation, with the last helical turn of the S4 segment relaxing R5 into alpha-helical form. In contrast, in the fully activated state, the region between R2 and R6

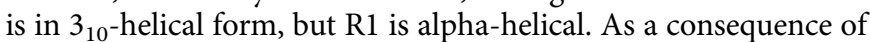
the $3_{10}$-helix conformation from R1 to R4 in the toxin/channel complex, the residues between R1-R2 and R3-R4 bridge the HCS such that R1-R2 and R3-R4 share the same vertical plane in their interactions with the negative side chains of the extracellular negative cluster (ENC) and intracellular negative cluster (INC), respectively. This unique linear voltage-sensor-trapped conformation would be strongly stabilized by these simultaneous gating charge interactions outside and inside the HCS, which may provide the chemical energy required for potent voltage-sensor trapping against the force of the transmembrane electrical field and therefore for effective modification of sodium channel gating. The potential gating charges R5 and R6 translocate to the intracellular side of the VS completely. These charged residues were proposed to interact with the CTD in the structure of the $\mathrm{Na}_{\mathrm{V}} \mathrm{Pas} /$ $\mathrm{Na}_{\mathrm{V}} 1.7$ chimera $^{26}$. However, the CTD was not resolved in our structure, preventing visualization of the potential binding positions of R5 and R6.

Superposition of the fully activated state (grey) and toxininduced intermediate-activated state (blue) of the DIV-VS revealed a remarkable conformational difference (Fig. 4c). From S1 through most of S3 there is little or no structural change, whereas the final two helical turns of S3 and the entire S4 segment undergo dramatic conformational shifts. Notably, Gly1607 serves as a pivot point for S3 rotation, and the rotation of upper S3, in turn, moves $\mathrm{S} 4$ downward $\sim 11 \AA$, such that R1 and R2 in the intermediate-activated state are approximately in the positions of R3 and R4 in the fully activated state (Fig. 4c). This toxin-induced conformational change in the S3-S4 linker is further documented by our fit to the cryo-EM density, which is illustrated in Supplementary Fig. 3c-e. At the intracellular end of S4, an elbowlike bend is formed between S4 and the S4-S5 linker, which pushes the S4-S5 linker $\sim 4.6 \AA$ inward at its N-terminal end (Fig. 4c). Intriguingly, our previous resting-state structure of $\mathrm{Na}_{\mathrm{V}} \mathrm{Ab}$ showed that a similar elbow pushes the S4-S5 linker and its connection to S4 strikingly inward and twists this segment in order to close the intracellular activation gate ${ }^{31}$. This conformational change in the S4-S5 linker is further supported by the close fit of our structural model to the cryo-EM density (Supplementary Fig. 4a). Superposition of the intermediate-activated DIV-VS structure (blue) upon the resting state $\mathrm{Na}_{V} \mathrm{Ab}$-VS structure (orange) further illuminates these conformational differences (Fig. 4d). The connecting S3-S4 loop of the intermediateactivated state of the LqhIII/ $/ \mathrm{Na}_{\mathrm{V}} 1.5_{\mathrm{C}}$ complex is not located as deeply inward as that of the resting-state of $\mathrm{Na}_{\mathrm{V}} \mathrm{Ab}$ and is not as tightly twisted (Fig. 4d). Moreover, the R1 and R2 gating charges are both located fully outward from the HCS in the partially activated S4 segment in the LqhIII/rNa $1.5_{\mathrm{C}}$ complex, whereas $\mathrm{R} 1$ is positioned only partially outward from the HCS in the resting state of $\mathrm{Na}_{\mathrm{V}} \mathrm{Ab}$ (Fig. 4d). In addition, the S4-S5 linker in the intermediate-activated state has not moved as deeply into the cytosol as in the resting state (Fig. 4d). These differences suggest that the toxin-induced intermediate-activated state of $\mathrm{Na}_{\mathrm{V}} 1.5 \mathrm{VS}$ is indeed an intermediate state between the resting state and the fully activated state.

A hallmark feature of the action of a-scorpion toxins is strongly voltage-dependent dissociation from their receptor site, which correlates with the voltage dependence of activation of sodium channels (Fig. 1c $c^{9,24,25}$ ). The structure of the $\mathrm{rNa}_{\mathrm{V}} 1.5_{\mathrm{C}} /$ LqhIII toxin complex reveals the molecular basis for this important aspect of scorpion toxin action. In the complex of the toxin with the partially activated state of the DIV-VS, the positive charge of the $\varepsilon$-amino group of K64 on LqhIII interacts with the same negatively charged side chain in the ENC that interacts with R1 and R2 in the activated conformation of the VS (Fig. 4a, b). In light of these structures, it seems likely that outward movement of the S4 segment during activation of the DIV-VS creates a clash with K64 and causes toxin dissociation by both electrostatic repulsion and steric hindrance. This potent combination of electrostatic repulsion and physical clash is sufficient to overcome the high binding energy of the a-scorpion toxins.

Partially open intracellular activation gate. Rapid voltagedependent activation is one of the signature functions of $\mathrm{Na}_{\mathrm{V}}$ channels ${ }^{1,2}$. A full understanding of the mechanism of activation requires structural information on $\mathrm{Na}_{\mathrm{V}}$ channels in different states. Side-by-side comparison of our previous activated-state structure with our current LqhIII-bound intermediate-activated structure of $\mathrm{rNa}_{\mathrm{V}} 1.5_{\mathrm{C}}$ reveals key steps in the coupling of the conformational changes of the DIV-VS to the intracellular activation gate (Figs. 4c and 5). To reach the intermediate-activated state (blue) from the fully activated state (grey), S4 of DIV-VS moves inward $\sim 11 \AA$ and pushes the DIV S4-S5 linker inward $\sim 4.6 \AA$ through the formation of an elbow-like bend (Figs. $4 \mathrm{c}$ and 5a). The shifted DIV S4-S5 linker engages DIV-S6 through Ser1655 (Figs. 4c and 5c). As a result, DIV-S6 shifts toward the pore axis, and this movement pushes DI-S6 away from the center of the orifice (Fig. 5a, b and Supplementary Fig. 4b, c). Meanwhile, the DI S4-S5 linker moves outward away from the activation gate, which shifts both DI-S6 and DII-S6 away from the 


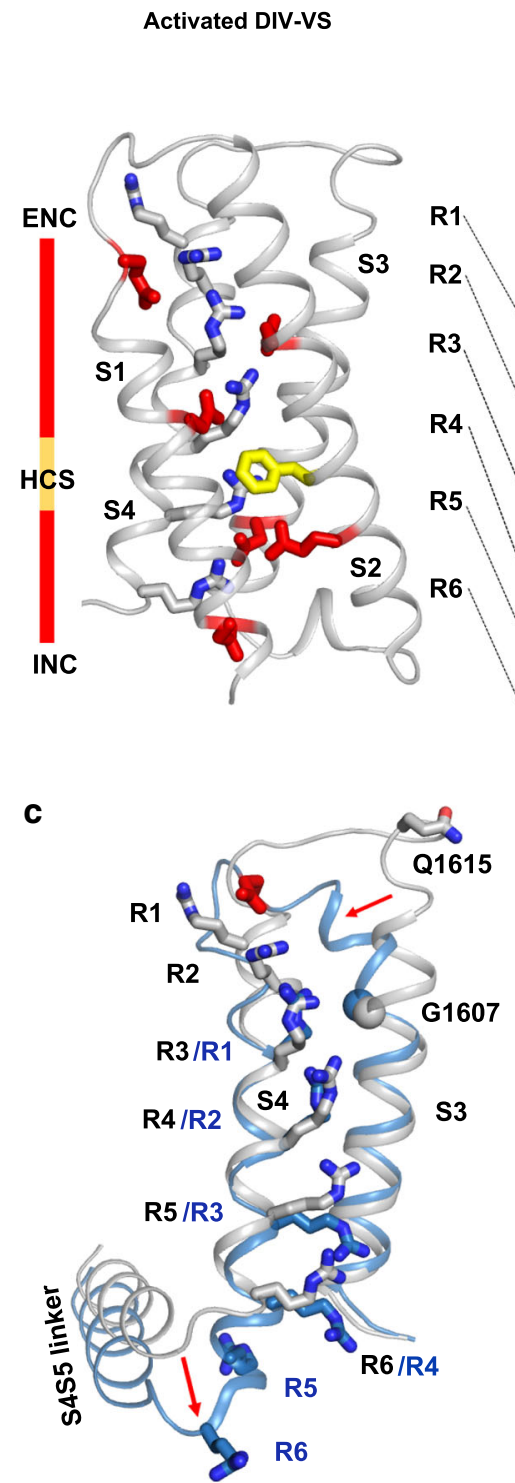

b

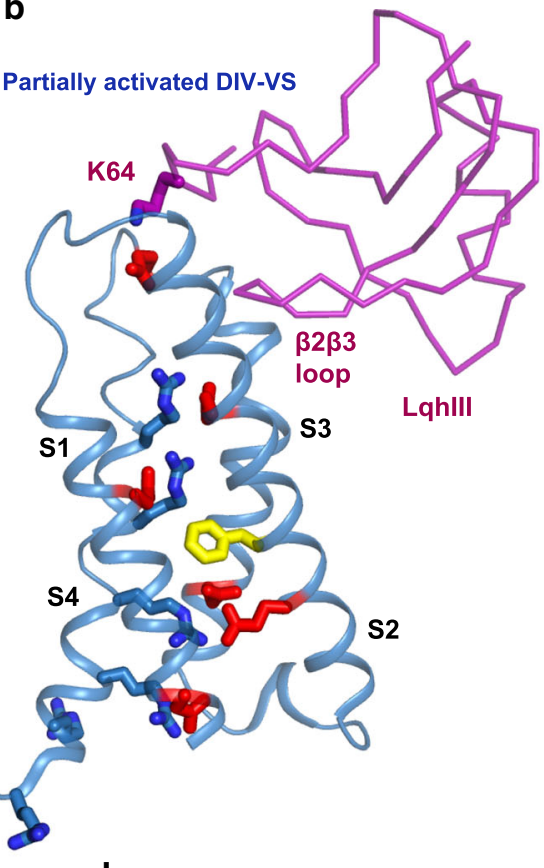

d

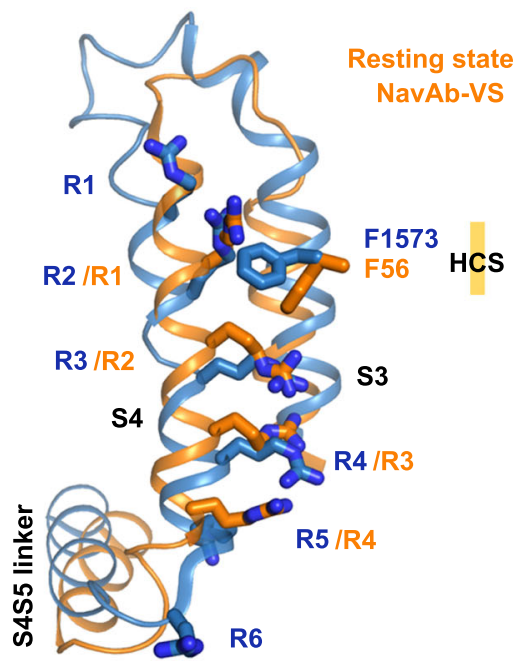

Fig. 4 Conformational Change of DIV-VS. a, b Structures of the activated Nav1.5 DIV-VS and intermediate-activated Nav1.5 DIV-VS were colored in grey and blue, respectively. Gating charges (grey or blue), ENC (red), HCS (yellow), and INC (red) were shown in sticks. The shift of each gating charge was indicated by black dashed lines. c Superposition of Nav1.5 DIV-VS between activated and intermediate-activated state. Red arrows indicate the conformational changes. d Superposition of the intermediate-activated Nav1.5 DIV-VS and resting-state NavAb-VS.

pore axis and contributes to a more open conformation. The shifts of the four S6 segments result in an enlarged activation gate with a van der Waals diameter of $6.6 \AA$ (Fig. 5b and Supplementary Fig. $4 b, c)$, which is $\sim 1 \AA$ larger than the activation gate in apo-rNa $1.5_{\mathrm{C}}$, but $\sim 2 \AA$ smaller than the expected orifice of the activation gate of fully open $\mathrm{rNa}_{\mathrm{V}} 1.5_{\mathrm{C}}$ when modeled using the open state of $\mathrm{Na}_{V} \mathrm{Ab}^{21,32}$. Unambiguous density shows Tyr1769 in DIV-S6 in an outward-pointing conformation making the opening of the activation gate larger, whereas it is pointed inward in the fully activated structure (Fig. 5b). Together, these conformational movements result in a wider opening in the activation gate in the toxin-induced intermediate-activated state, which could in principle be sufficient for sodium conductance.

Consistent with the more open conformation of the activation gate, we observed a much stronger density of bound lipid or detergent in the lumen of the activation gate in our intermediate activated $\mathrm{rNa}_{\mathrm{V}} 1.5_{\mathrm{C}}$ structure compared to that of the activated
apo-rNa $1.5_{\mathrm{C}}$ structure (Supplementary Fig. $4 \mathrm{~b}, \mathrm{c}$ ). Considering that the two proteins were purified following the same procedures, it is likely that the same set of lipid and detergent molecules would be available for binding. Therefore, we believe the larger lipid or detergent molecule is able to bind in the lumen of the activation gate of the intermediate-activated structure because of its larger diameter rather than because of a change in lipid or detergent concentrations between the two protein preparations.

Loosely bound fast inactivation gate. In activated $\mathrm{Na}_{\mathrm{V}}$ structures, the IFM motif of the fast inactivation gate binds to a hydrophobic pocket formed by DIII S4-S5 linker and the intracellular ends of DIV-S5 and DIV-S6 ${ }^{19-21}$. In the intermediateactivated $\mathrm{rNa}_{\mathrm{V}} 1.5_{\mathrm{C}}$ /LqhIII structure, weak, yet consistent, density for the IFM motif suggests that the conformational changes of the VS and activation gate have in turn altered the conformation of 
a

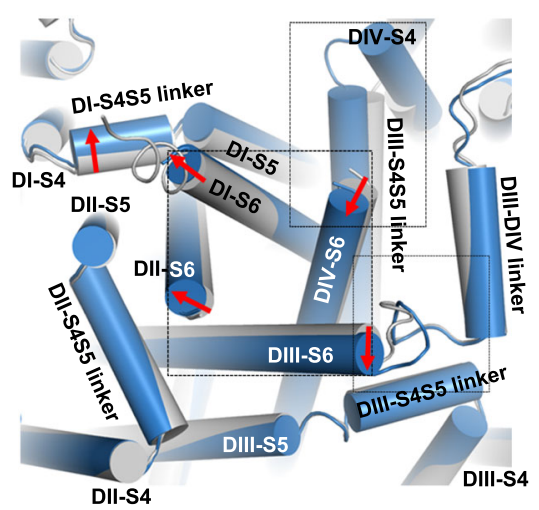

C

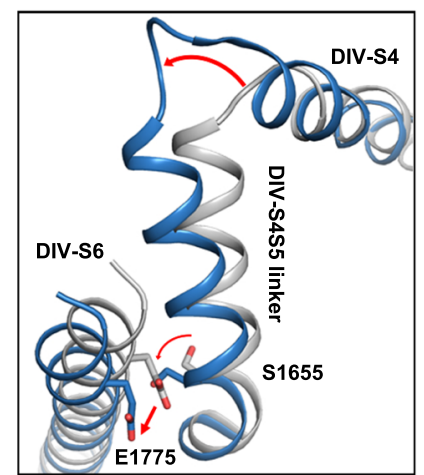

b

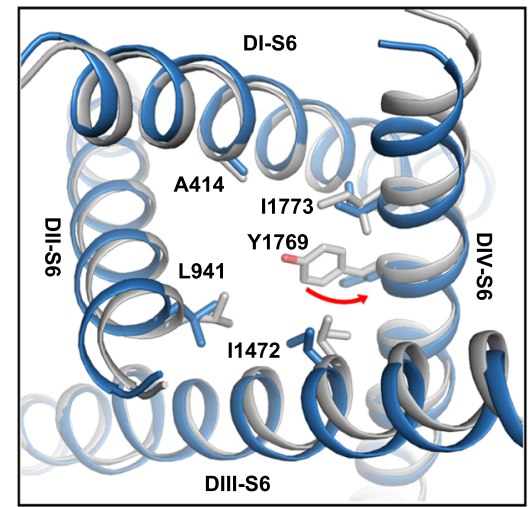

d

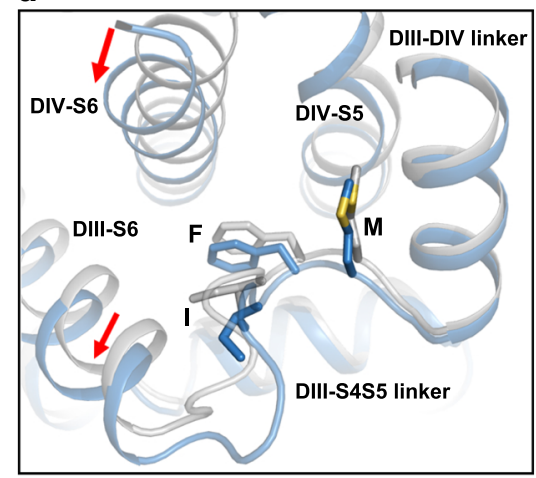

$\mathbf{e}$
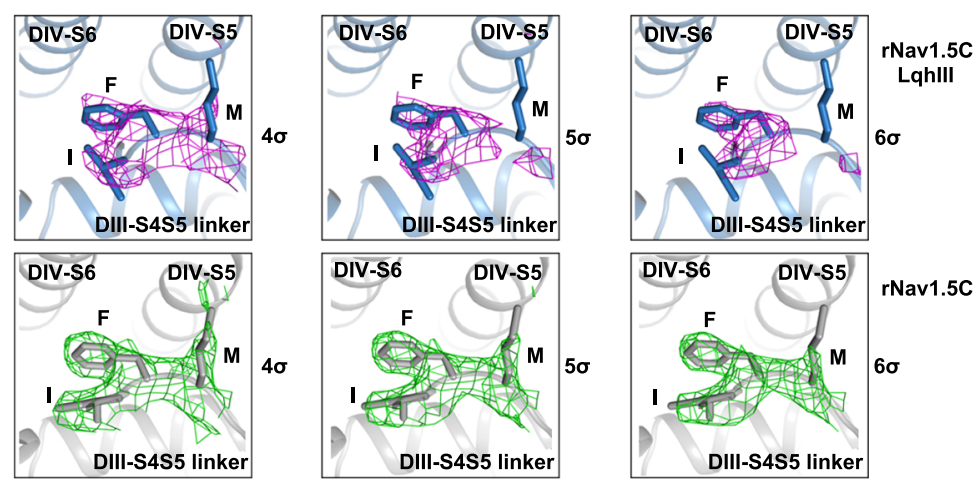

Fig. 5 Coupling of LqhIII binding and conformational change in DIV-VS to the S4-S5 linkers and the intracellular activation gate. a Superposition of Nav1.5 intracellular gate between activated (grey) and intermediate-activated (blue) states of the DIV-VS. Red arrows indicate the conformational changes. The black dash squares indicated for panels (b-d). b Zoom-in view of the intracellular activation gate with constriction residues shown in sticks. $\mathbf{c}$ Zoom-in view of DIV S4-S5 linker mediates the conformational changes. $\mathbf{d}$ Zoom-in view of IFM motif shift between activated and deactivated state. e Close-up views of the IFM motif in the free and LqhIII-complexed Nav1.5 together with its cryo-EM density contoured at different $\sigma$ levels.

the bound IFM motif and made it less stable (Fig. 5d, e, Supplementary Fig. $4 \mathrm{~b}, \mathrm{c})$. Evidently, the movement of DIV-S6 squeezes the binding pocket, and the shift of DIII-S6 destabilizes binding of the IFM motif. We observed a small shift in the position of the IFM motif in the intermediate-activated structure, as well as weaker density for the Met side chain, consistent with greater mobility and partial dissociation (Fig. 5d, e). These changes are illustrated most clearly in Fig. 5e by comparing cryoEM density contoured at $4 \sigma, 5 \sigma$, and $6 \sigma$ in the presence and absence of LqhIII. We propose that $\alpha$-scorpion toxins inhibit fast inactivation by both trapping the DIV-VS in an intermediateactivated conformation and altering the shape of the IFM binding pocket, which causes slowed IFM binding, more rapid dissociation of the IFM motif, and release of the fast inactivation gate. These effects are responsible for the slowed rate of fast inactivation and the incomplete extent of fast inactivation that are hallmarks of the action of LqhIII and other a-scorpion toxins (Fig. 1).

LqhIII does not open the intracellular activation gate. Our static cryo-EM view of the intracellular activation gate of apo$\mathrm{rNa}_{\mathrm{V}} 1.5_{\mathrm{C}}$ suggests that it is partially open compared to our tightly closed resting-state model of $\mathrm{rNa}_{\mathrm{V}} 1.5_{\mathrm{C}}$ based on the $\mathrm{Na}_{\mathrm{V}} \mathrm{Ab}$ resting state ${ }^{21,31}$. However, it does not appear to be open enough to conduct hydrated $\mathrm{Na}^{+} 32,33$. To test this hypothesis, we used molecular dynamics methods similar to those we previously applied to the $\mathrm{Na}_{\mathrm{V}} \mathrm{Ab}$ structure ${ }^{32,33}$ in order to investigate the effect of LqhIII on pore hydration and dilation of the intracellular activation gate (Fig. 6). The inner pore of $\mathrm{rNa}_{\mathrm{V}} 1.5_{\mathrm{C}}$ is depicted 

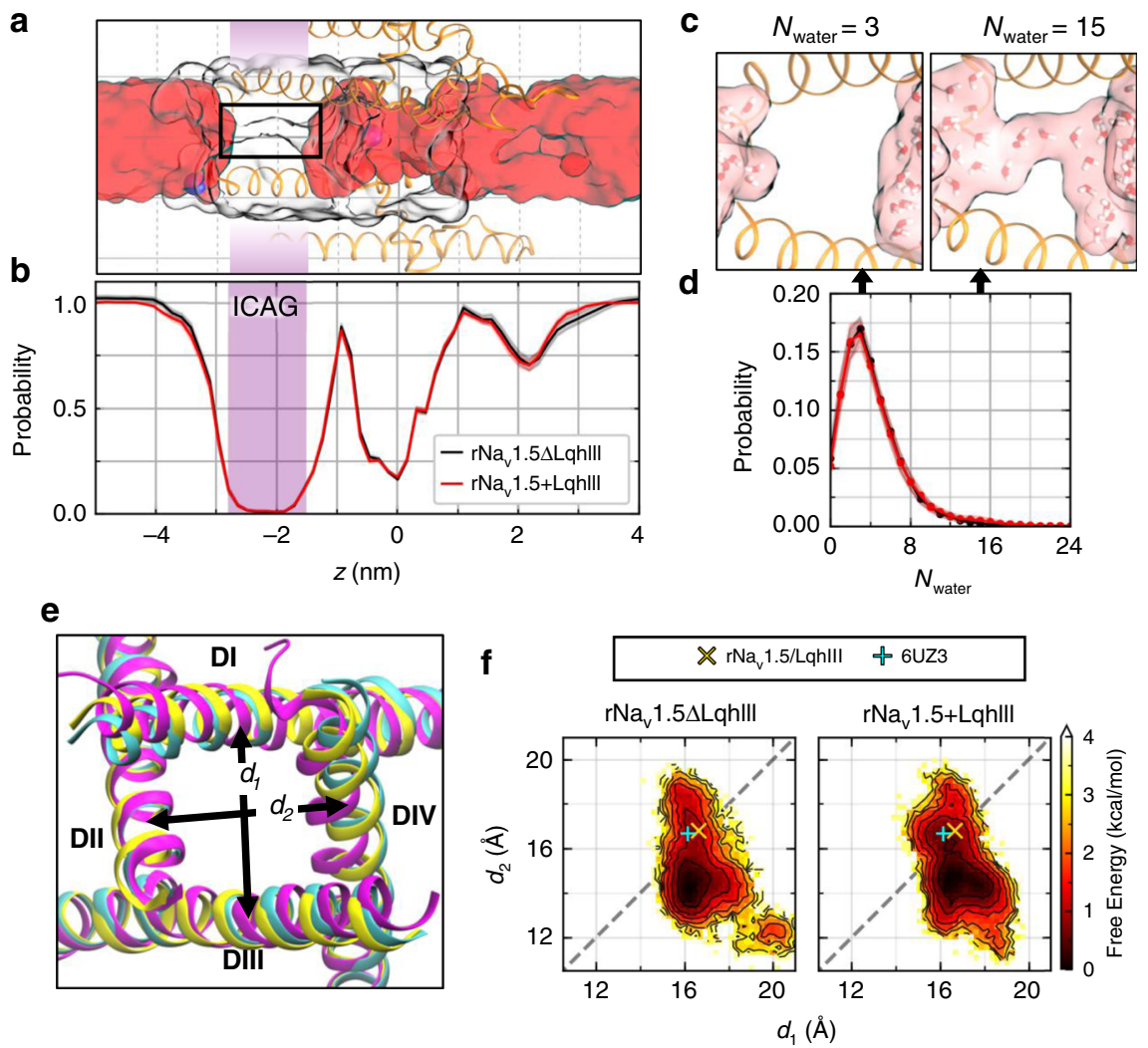

Fig. 6 Molecular dynamics analysis of hydration and $\mathbf{N a}{ }^{+}$permeation through the rNav1.5c/LqhIII complex. a Side view of $r$ Nav $1.5_{C}$ (orange ribbons; domains II and IV) from MD simulations highlighting $\mathrm{Na}^{+}$ions (blue spheres), the water-occupied volume within a cylinder of radius $8.5 \AA$ (red surface), and the protein-occupied volume within a cylinder of radius $12 \AA$ (colorless surface). In this snapshot, the protein cavity (outlined in a black rectangle) at the intracellular activation gate (ICAG; purple shaded region, $-2.8 \mathrm{~nm}<z<-1.5 \mathrm{~nm}$ ) is dehydrated. The QuickSurf representation in VMD was used for surfaces. b Average hydration along the pore-axis for simulations of $r \mathrm{Nav}_{\mathrm{v}} 1.5 \mathrm{C} /$ LqhIII (red line) with and (black line) without the toxin. Overall pore hydration was unchanged whether or not the toxin is included in simulations. Shading corresponds to the standard error of the mean (s.e.m.). c Molecular representations of the gate containing $N_{\text {water }}=3$ (left) or 15 (right) water molecules. d Average probability distribution of $N_{\text {water }}$ in the gate. The gate is more likely to contain 10 or more water molecules when the toxin is present. Data points and shading represent mean and s.e.m. e Bottom (intracellular) view of the activation gate for cryo-EM rNav1.5 (PDB ID: 6UZ3; cyan), cryo-EM rNav1.5c/LqhIII (yellow), and an example conformation from the most sampled basin in MD (magenta) are superimposed. The distances $d_{1}, d_{2}$ between opposing helices are shown schematically (see "Methods"). $\mathbf{f}$ Free energy of $d_{1}$ vs. $d_{2}$ computed from MD simulations. The reference cryo-EM structures of (cyan + ) rNav $1.5_{C}$ and (yellow $\times$ ) rNav $1.5 \mathrm{C} /$ Lqhlll are indicated. Contour lines are shown every $0.5 \mathrm{kcal} / \mathrm{mol}$ from 0 to $4 \mathrm{kcal} / \mathrm{mol}$. In the simulations, the intracellular activation gate often contracts and adopts an asymmetric conformational basin with $d_{2}<d_{1}$, with the symmetric conformation observed by cryo-EM corresponding to a metastable state 0.5 to $1 \mathrm{kcal} / \mathrm{mol}$ higher in free energy. Time frames spread across 30 independent simulations of $r N a v 1.5 \mathrm{C}$ with or without LqhIII were used for analyses $(n=29,026$ frames for $r \mathrm{Na}_{\mathrm{v}} 1.5_{\mathrm{C}}$ and $n=29,899$ frames for $\mathrm{rNa} \mathrm{v}_{\mathrm{V}} 1.5_{\mathrm{C}}$ /LqhIII).

lying from the right (extracellular) to the left (intracellular) with the surrounding S5 and S6 helices illustrated in orange (Fig. 6a). Water molecules (red) fill the inner part of the central cavity on the right and the intracellular exit from the pore on the left. However, in this snapshot, there is a gap in hydration in the intracellular activation gate itself (white), where the S6 segments come together in a bundle (orange helices, Fig. 6a). In fact, statistical analysis of the conformational ensemble shows that the average probability density of water molecules in the intracellular activation gate (purple band) is near zero in simulations of both $\mathrm{rNa}_{\mathrm{V}} 1.5_{\mathrm{C}}$ (black) and $\mathrm{rNa}_{\mathrm{V}} 1.5_{\mathrm{C}} /$ LqhIII (red; Fig. 6b). Accordingly, $\mathrm{Na}^{+}$did not permeate through the dehydrated activation gate in any of the simulations, suggesting that the pore is functionally closed. Not only is the activation gate the least hydrated region of the pore on average, but it is also nearly always dehydrated (Fig. 6a, b). Even when a pathway connecting the central cavity to the intracellular space is transiently present, water molecules are usually excluded from entering this region due to the hydrophobic effect (Fig. 6a). As such, the activation gate is predominantly dehydrated (dewetted) and occupied by 4 water molecules on average, compared to $10-11$ molecules of water in the open activation gate of $\mathrm{Na}_{\mathrm{V}} \mathrm{Ab}^{32}$. Thus, the intracellular activation gate of $\mathrm{rNa}_{\mathrm{V}} 1.5_{\mathrm{C}}$, much like that of other voltage-gated ion channels, fits the paradigm of a hydrophobic activation gate, where small increases in the size of the gate tilt the wetted/ dewetted equilibrium towards the wetted state as a prerequisite to ion permeation ${ }^{32,34-37}$. These results showing that the intracellular activation gate is functionally closed in the $\mathrm{rNa}_{\mathrm{V}} 1.5_{\mathrm{C}} / \mathrm{LqhIII}$ complex illustrate the structural basis for the well-established effect of $\alpha$-scorpion toxins to slow and prevent fast inactivation of sodium channels without opening the pore and allowing sodium conductance.

Fluctuations of the diameter of the activation gate. The distances between opposing S6 helix tails $\left(d_{1}: D I-D I I I, d_{2}: D I I-D I V\right)$ were monitored to estimate the dilation of the gate (Fig. 6e, f). The activation gate in the cryo-EM structure of $\mathrm{rNa}_{\mathrm{V}} 1.5_{\mathrm{C}} / \mathrm{LqhIII}$ is slightly wider and more symmetric $\left[\left(d_{1}, d_{2}\right)=(16.6 \AA, 16.8 \AA)\right]$ than that of the cryo-EM structure of $\mathrm{rNa}_{\mathrm{V}} 1.5_{\mathrm{C}}\left[\left(d_{1}, d_{2}\right)=(16.1 \AA, 16.6 \AA)\right.$; Fig. $6 \mathrm{e}, \mathrm{f}]^{21}$. In the simulations, the structure of the gate fluctuates, 
with $d_{2}$ deviating by up to $4 \AA$ from the toxin-bound cryo-EM structure (Fig. 6f). Overall, asymmetric conformations in which $d_{2}<$ $d_{1}$ are slightly favored (by $0.5-1.0 \mathrm{kcal} / \mathrm{mol}$ ) relative to the symmetric cryo-EM conformation of $\mathrm{rNa}_{\mathrm{V}} 1.5_{\mathrm{C}} / \mathrm{Lqh}$ III. The average values of both $d_{1}$ and $d_{2}$ undergo small but significant increases relative to simulations without LqhIII, as does the average number of water molecules in the gate (Supplementary Fig. 5a). Although the pore remains predominantly dewetted irrespective of the presence of LqhIII, fluctuations leading to more dilated conformations of the activation gate are correlated with larger hydration numbers (Supplementary Fig. 6a), with transient occurrences of 10 or more water molecules connecting the central cavity to the intracellular environment (Fig. 6c, d). However, the activation gate is not significantly more likely to be wetted in simulations of $\mathrm{rNa}_{\mathrm{V}} 1.5_{\mathrm{C}}$ with LqhIII than in simulations without LqhIII (Supplementary Fig. 5b), consistent with the fact that toxin binding does not open the gate sufficiently for passage of $\mathrm{Na}^{+}$. Nevertheless, the analysis of fluctuations in diameter and hydration of the intracellular activation gate provides an initial suggestion that LqhIII binding may facilitate the transition to the open state of $\mathrm{Na}_{\mathrm{V}} 1.5$, which requires activation of the VSs in domains I-III for completion.

\section{Discussion}

We determined the structure of rat $\mathrm{Na}_{\mathrm{V}} 1.5_{\mathrm{C}}$ in complex with the a-scorpion toxin LqhIII by single-particle cryo-EM. Biochemical and biophysical studies support only a single neurotoxin receptor site 3 per sodium channel located in the VS in DIV, at which ascorpion toxins, sea anemone toxins, and related gating-modifier toxins bind ${ }^{9,10}$. Consistent with this expectation from functional studies, we found a single molecule of LqhIII bound to the VS in $D I V$. The toxin binds at the extracellular end of the aqueous cleft formed by the S1-S2 and S3-S4 helical hairpins in the VS through its $\beta 2 \beta 3$ loop and its C-terminal. Many conserved amino acid residues that are important for $\alpha$-scorpion toxin binding and its functional effects on sodium channels are located in key positions in the toxin-receptor-binding interface (Fig. 3e). These results provide convincing evidence that we have correctly identified the pharmacologically important Neurotoxin Receptor Site 3 on the native cardiac sodium channel and defined its mode of toxin binding at high resolution. a-Scorpion toxins bind to Neurotoxin Receptor Site 3 in a voltage-dependent manner, with high-affinity binding to the resting state $e^{9,24,25}$. Depolarization reduces toxin affinity and causes toxin dissociation ${ }^{9,24,25}$. The bound toxin prevents the normal outward movement of the gating charges in DIV-S4, as measured directly from gating currents ${ }^{10}$. The outward movement of the DIV-S4 segment correlates with fast inactivation, as measured by voltage clamp fluorometry with specifically labeled $S 4$ residues ${ }^{12,13}$. These studies highlight the importance of DIV-S4 and neurotoxin receptor site 3 in triggering fast inactivation. LqhIII prefers to bind to the resting state of $\mathrm{rNa}_{\mathrm{V}} 1.5_{\mathrm{C}}$, and strong depolarization causes dissociation in biochemical and electrophysiological studies using nM concentrations of toxin (Fig. 1) ${ }^{9,24,25}$. In spite of that, the binding affinity of LqhIII for $\mathrm{rNa}_{\mathrm{V}} 1.5_{\mathrm{C}}$ is high enough to overcome this opposing electrostatic energy gradient when high concentrations of $\mathrm{rNa}_{\mathrm{V}} 1.5_{\mathrm{C}}$ and LqhIII are used to drive the binding interaction and induce gating charge transfer of DIV-VS into the intermediate-activated state. The S4 segment of the intermediate-activated DIV-VS has shifted two helical turns inward when compared with that of activated DIV-VS; however, it is not as deeply inward as in the resting state of the $\mathrm{Na}_{\mathrm{V}} \mathrm{Ab}-\mathrm{VS}$, which suggests the toxin-induced intermediate-activated state is indeed a normal intermediate state in the function of the VS. To account for the functional effects of the toxin, the toxin-modified VS must allow activation and pore opening, even while impairing fast inactivation. Trapping the DIV-VS in an intermediateactivated state that does not trigger fast inactivation, but nevertheless is permissive for activation driven by the other three VS, would satisfy this mechanistic requirement. Thus, we propose that the toxin-induced intermediate-activated state-defined here is indeed the voltage-sensor trapped state that is responsible for the gating-modifier properties of the a-scorpion toxins. The Binding of LqhIII traps the VS in a unique conformation. The S4 segment is in $3_{10}$ helical conformations from R1 to R4, with two gating charges on each side of the HCS (Fig. 4). This conformation places the four gating charges in a straight line. R1 and R2 interact strongly with two negatively charged side chains of the ENC, whereas R3 and R4 interact strongly with two negatively charged side chains in the INC. These four ion-pair interactions bridging the HCS are unique in VS function. No other position of the primary gating charges can simultaneously form four ion-pair interactions bridging the HCS. We propose that this unique, toxin-stabilized conformation of the gating charges is the key voltage-sensor-trapped state that allows the deathstalker scorpion toxin to paralyze and kill its prey. The movement of the S4 segment implied by comparison of the structures of apo$\mathrm{Na}_{\mathrm{V}} 1.5_{\mathrm{C}}$ and LqhIII/ $\mathrm{rNa}_{\mathrm{V}} 1.5_{\mathrm{C}}$ fits closely with the sliding-helix model of voltage sensing ${ }^{31,38,39}$. Compared to the activated state, in the toxin-induced intermediate-activated state, the S4 segment moves inward $\sim 11 \AA$ and rotates slightly as $3_{10}$ helix is converted to alpha-helix on the intracellular side of the HCS. The gating charges exchange ion-pair partners from the ENC to the INC; however, they do not move as far inward as observed in the resting state. Thus, the toxin-induced conformational change in the VS exactly follows the proposed voltage-driven movement of the S4 segment based on the sliding helix voltage-sensing model and the structures of the activated and resting states of $\mathrm{Na}_{\mathrm{V}} \mathrm{Ab}^{31}$.

Our complex structure provides an excellent model for investigating the coupling between gating charge transfer and fast inactivation. The cryo-EM structure of $\mathrm{AaHII} / \mathrm{Na}_{\mathrm{V}} \mathrm{Pas}-\mathrm{Na}_{\mathrm{V}} 1.7-$ DIV-VS chimera suggested that R5 and K6 of $\mathrm{Na}_{\mathrm{V}} 1.7-D I V-V S$ were stabilized by interaction with the $\mathrm{Na}_{\mathrm{V}} \mathrm{Pas}$ CTD. By contrast, in our fully functional LqhIII/ $\mathrm{rNa}_{\mathrm{V}} 1.5_{\mathrm{C}}$ structure, the CTD was not observed. In fact, the CTD's also were not observed in the high-resolution structures of human $\mathrm{Na}_{\mathrm{V}} 1.2,1.4$, and 1.7 channels either ${ }^{19-21,40}$. These results suggest that the CTDs of native mammalian $\mathrm{Na}_{V}$ channels are disordered and/or mobile and differ substantially from the cockroach CTD in the $\mathrm{Na}_{V} \mathrm{Pas}$ $\mathrm{Na}_{V} 1.7$ chimera, whose amino acid sequence is not similar to the CTD's of mammalian $\mathrm{Na}_{\mathrm{V}}$ channels. Based on this comparison, it seems likely that the CTD plays a secondary role or a regulatory role in fast inactivation in mammalian $\mathrm{Na}_{\mathrm{V}}$ channels, which may include transient interactions with the potential R5 and R6 gating charges of DIV-S4. Our structure gives additional insights into the structural basis for coupling of the conformational change of the intermediate-activated DIV-VS to fast inactivation. The inward movement of the DIV-S4 segment and its gating charges from their activated positions propagates a voltage-driven conformational change inward to the DIV S4-S5 linker, forming an elbow that angles the S4-S5 linker into the cytosol. This substantial movement twists the S4-S5 linker and disturbs the conformation of the binding pocket for the IFM motif, thereby destabilizing the fast-inactivated state. In contrast, the forward coupling of VS movement to fast inactivation likely involves outward movement of the S4 segment, unbending the elbow, untwisting the S4-S5 linker, and opening the receptor site for binding of the IFM motif of the fast inactivation gate. Binding of LqhIII would oppose this series of conformational events that lead to fast inactivation. Compared to the resting state, the outward movement of the DIV-S4 segment in the partially activated VS and the resulting loosening of the elbow bend in the DIV 
S4-S5 linker also partially opens the activation gate through the coupled movement of the nearby intracellular end of DI-S6 away from the pore axis. Our molecular dynamics analyses show that this partially open activation gate structure is still functionally closed with respect to $\mathrm{Na}^{+}$conductance. Nevertheless, this conformational change may be an essential permissive movement of $D I V$ that allows subsequent activation of the DI-DIII VSs to drive pore opening with only partial activation of the DIV VS in its toxin-induced intermediate-activated state. This coupling among domains may occur through the domain-swapped organization that places the DIV-VS adjacent to the DI-PM. Consistent with this idea, the DI-S6 segment is moved away from the axis of the pore in the toxin-induced deactivated state, similar to its movement during the pore opening of $\mathrm{Na}_{\mathrm{V}} \mathrm{Ab}$. In this position, it would promote activation and pore opening of DII-DIV activation gate residues to give the fully open channel.

In previous work, the structure of a chimera of the cockroach sodium channel $\mathrm{Na}_{\mathrm{V}} \mathrm{Pas}$ with the AaHII toxin bound was determined by cryo- $\mathrm{EM}^{26}$. The functional significance of this sodium channel in the cockroach is unknown, and this chimera containing a segment of the DIV-VS of human $\mathrm{Na}_{\mathrm{V}} 1.7$ was nonfunctional; therefore, it is difficult to precisely compare this prior work to the structures we present here. Unexpectedly, AaHII bound to the $\mathrm{Na}_{\mathrm{V}} \mathrm{Pas}$ chimera in two positions, one on the VS in $D I$ of $\mathrm{Na}_{\mathrm{V}} \mathrm{Pas}$ and one on the DIV-VS contributed in part by $\mathrm{Na}_{\mathrm{V}} 1.7$, and it was not shown whether either of these sites was functional in the chimera ${ }^{26}$. In contrast, we found only a single toxin binding site, as expected from previous structure-function studies ${ }^{9,10}$. Neurotoxin Receptor Site 3 identified in our study is generally similar to the AaHII binding site found in DIV of the AaHII/Na $\mathrm{Nas}_{\mathrm{P}} \mathrm{Na}_{\mathrm{V}} 1.7-\mathrm{DIV}$-VS chimera structure ${ }^{26}$, but we found an important difference in the binding poses of the two toxins. Compared with AaHII bound to the $\mathrm{Na}_{\mathrm{V}} \mathrm{Pas}_{\mathrm{Na}} \mathrm{Na}_{\mathrm{V}} 1.7-\mathrm{DIV}$ VS chimera, LqhIII bound to $\mathrm{rNa}_{\mathrm{V}} 1.5_{\mathrm{C}}$ is rotated $\sim 26^{\circ}$ downward, further away from the glycan and DI of the channel (Supplementary Fig. 7). This difference may reflect an alteration

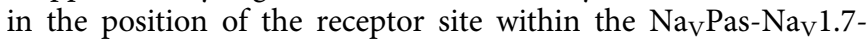
DIV-VS chimera tertiary structure caused by artifactual constraints from the formation of the chimera ${ }^{26}$. Alternatively, the structure of the functionally active LqhIII/ $/ \mathrm{Na}_{\mathrm{V}} 1.5_{\mathrm{C}}$ complex described here may be characteristic of the cardiac sodium channel, which has numerous distinct features compared to neuronal sodium channels like $\mathrm{Na}_{\mathrm{V}}$ 1.7. The exact position and composition of the glycan moiety adjacent to Neurotoxin Receptor Site 3 in $\mathrm{rNa}_{\mathrm{V}} 1.5_{\mathrm{C}}$ may be one potentially important point of difference. Despite this difference in the position of the bound toxin, the overall structures of the two toxin-bound DIVVSs are remarkably similar with an RMSD of $1.41 \AA$ over 112 residues. This striking structural similarity indicates that the DIVVS's in these two channel constructs are locked in a similar state by toxin binding at $0 \mathrm{mV}$.

Sea anemone toxins are not similar to scorpion toxins in amino acid sequence, yet they bind to Neurotoxin Receptor Site 3 and inhibit fast inactivation like $\alpha$-scorpion toxins ${ }^{9,41}$. Some sea anemone toxins, like Anthopleurin A and B, are highly active on cardiac sodium channels ${ }^{42,43}$. It is likely that the sea anemone toxins trap the DIV-VS in a similar intermediate-activated conformation as we have observed here for LqhIII. $\beta$-Scorpion toxins are similar in structure to $\alpha$-scorpion toxins ${ }^{44}$. They bind to Neurotoxin Receptor Site 4, which is located in the DII-VS in an analogous position to Neurotoxin Receptor Site 3 in the DIV$\mathrm{VS}^{45-47}$. They bind with high affinity to the activated state and trap the DII-VS in its outward, activated position ${ }^{45}$. This mode of voltage-sensor trapping enhances activation by shifting its voltage dependence to more negative membrane potentials ${ }^{45}$. When they act synergistically in scorpion venom, $\beta$ - and $\alpha$-scorpion toxins negatively shift the voltage dependence of activation and block fast inactivation, respectively, resulting in persistently activated sodium channels, repetitive firing, depolarization block of neuromuscular transmission, and lethal arrhythmias in the heart. A large family of cysteine-knot toxins from spiders also act as gating modifiers of voltage-gated ion channels ${ }^{48}$. They bind to the VS of $\mathrm{Na}_{V}, \mathrm{~K}_{V}$, and $\mathrm{Ca}_{\mathrm{V}}$ channels in an analogous manner to $a$-scorpion toxins and either block or enhance activation of the VS. The spider toxins that enhance activation may also stabilize the unique voltage-sensor-trapped conformation of the VS that we have elucidated here, in which a linear configuration of the four primary gating charges bridges the HCS and interacts simultaneously with the ENC and INC in a high-affinity voltage-sensortrapped complex. Thus, the toxin-bound state we have characterized here may have broad significance for VS trapping by a wide range of gating-modifier toxins from hundreds of species of spiders, scorpions, mollusks, and coelenterates, which all use this universal mechanism to immobilize their prey.

\section{Methods}

Electrophysiology. All experiments were performed at room temperature $\left(21-24^{\circ} \mathrm{C}\right.$ ) as described previously ${ }^{21}$. Human HEK293S $\mathrm{GnTI}^{-}$cells (American Type Culture Collection Cat\# CRL-3022) were maintained and infected on cell culture plates in Dulbecco's Modified Eagle Medium (DMEM) supplemented with $10 \%$ fetal bovine serum and glutamine/penicillin/streptomycin at $37^{\circ} \mathrm{C}$ and $5 \%$ $\mathrm{CO}_{2}$ for electrophysiology. Unless otherwise mentioned, HEK293S $\mathrm{GnTI}^{-}$cells were held at $-120 \mathrm{mV}$ and $100-\mathrm{ms}$ pulses were applied in $10 \mathrm{mV}$ increments from $-120 \mathrm{mV}$ to $+60 \mathrm{mV}$. A P/-4 holding leak potential was set to $-120 \mathrm{mV}$. Extracellular solution contained in mM: $140 \mathrm{NaCl}, 2 \mathrm{CaCl}_{2}, 2 \mathrm{MgCl}_{2}, 10 \mathrm{HEPES}, \mathrm{pH} 7.4$ Intracellular solution contained: $35 \mathrm{NaCl}, 105 \mathrm{CsF}, 10$ EGTA, 10 HEPES, pH 7.4 Glass electrodes had a resistance of 1.5-3 $\mathrm{M} \Omega$. Currents resulting from applied pulses were filtered at $5 \mathrm{kHz}$ with a low-pass Bessel filter and then digitized at $20 \mathrm{kHz}$. Data were acquired using an Axopatch 200B amplifier (Molecular Devices). Voltage commands were generated using Pulse 8.5 software (HEKA, Germany) and ITC18 analog-to-digital interface (Instrutech, Port Washington, NY).

Protein expression and purification. Detailed expression and purification of rat $\mathrm{rNa}_{\mathrm{V}} 1.5_{\mathrm{C}}$ were described in our previous study ${ }^{21}$. Briefly, $\mathrm{rNa}_{\mathrm{V}} 1.5_{\mathrm{C}}$ was expressed in HEK293S GnTI- cells (ATCC). The protein was extracted by $1 \%(\mathrm{w} / \mathrm{v}) \mathrm{n}$ dodecyl- $\beta$-D-maltopyranoside (DDM, Anatrace) and $0.2 \%(\mathrm{w} / \mathrm{v})$ cholesteryl hemisuccinate Tris salt (CHS, Anatrace) in Buffer A containing $25 \mathrm{mM}$ HEPES $\mathrm{pH}=7.4,150 \mathrm{mM} \mathrm{NaCl}$ and $10 \%$ glycerol. After centrifugation, the supernatant was agitated with anti-Flag M2-agarose resin (Sigma). Flag resin was washed in Buffer A supplemented with $0.06 \%$ glycol-diosgenin (GDN, Anatrace). Purified protein was then loaded onto a Superose- 6 column (GE Healthcare) in $20 \mathrm{mM}$ HEPES $\mathrm{pH}=7.4,150 \mathrm{mM} \mathrm{NaCl}$ and $0.06 \%$ GDN, peak fractions were concentrated to $\sim 1 \mathrm{mg} / \mathrm{ml}$ and mixed with $50 \mu \mathrm{M}$ LqhIII (Latoxan Laboratory) and purified FGF12b and calmodulin overnight. The mixture was then re-loaded to Superose- 6 column pre-equilibrated with buffer containing $25 \mathrm{mM}$ imidazole $\mathrm{pH}=6.0,150 \mathrm{mM} \mathrm{NaCl}$ and $0.006 \% \mathrm{GDN}$. Finally, peak fractions were concentrated to $40 \mu \mathrm{l}$ at $5 \mathrm{mg} / \mathrm{ml}$.

CryoEM grid preparation and data collection. Three microliters of purified sample were applied to glow-discharged holey gold grids (UltraAuFoil, 300 mesh, R1.2/1.3), and blotted for $2.0-3.5 \mathrm{~s}$ at $100 \%$ humidity and $4{ }^{\circ} \mathrm{C}$ before being plunged frozen in liquid ethane cooled by liquid nitrogen using an FEI Mark IV Vitrobot. All data were acquired using a Titan Krios transmission electron microscope operated at $300 \mathrm{kV}$, a Gatan K2 Summit direct detector, and Gatan Quantum GIF energy filter with a slit width of $20 \mathrm{eV}$. A total of 4222 movie stacks were automatically collected using Leginon ${ }^{49}$ at a nominal magnification of $130,000 \times$ with a pixel size of $0.528 \AA$ (super-resolution mode). Defocus range was set between -1.2 and $-2.8 \mu \mathrm{m}$. The dose rate was adjusted to 8 counts/pixel/s, and each stack was exposed for $8.4 \mathrm{~s}$ with 42 frames with a total dose of $60 \mathrm{e}^{-/} \AA^{2}$.

Cryo-EM data processing. The movie stacks were motion-corrected with MotionCorr $2^{50}$, binned 2-fold, and dose-weighted, yielding a pixel size of $1.056 \AA$. Defocus values of each aligned sum were estimated with Gctf $^{51-53}$. A total of 3805 micrographs with CTF fitted better than $6 \AA$ were used for particle picking, and a total of $1,817,940$ particles were automatically picked in RELION3. ${ }^{51}$. After several rounds of 2D classification, 882,608 good particles were selected and subjected to one class global angular search $3 \mathrm{D}$ classification with an angular search step at $7.5^{\circ}$, at which a low-pass filtered cryo-EM map of $\mathrm{rNa}_{\mathrm{V}} 1.5_{\mathrm{C}}$ was used as an initial model. Each of the last five iterations was further subjected to four classes of local angular search and $3 \mathrm{D}$ classification with an angular search step at $3.75^{\circ}$. After 
combining particles from the best $3 \mathrm{D}$ classes and removing duplicate particles, 570,843 particles were subjected to per-particle CTF estimation by GCTF followed by Bayesian polishing. The polished particles were subjected to last round threeclass multi-reference 3D classification. The best class containing 267,595 particles was auto-refined and sharpened in Relion3.0. Local resolution was estimated by ResMap in Relion3.0. A diagram illustrating our data processing is presented in Supplementary Fig. 2.

Model building and refinement. The structures of rat $\mathrm{rNa}_{\mathrm{V}} 1.5_{\mathrm{C}}$ (PDB code: 6UZ0) and LqhIII (PDB code: 1FH3) were fitted into the cryo-EM density map in Chimera $^{52}$. The model was manually rebuilt in COOT $^{54}$ and subsequently refined in Phenix ${ }^{55}$. The model vs. map FSC curve was calculated by Phenix.mtrage. Statistics for cryo-EM data collection and model refinement are summarized in Supplementary Table 1 .

Molecular dynamics model. The cryo-EM structure of $\mathrm{rNa}_{\mathrm{V}} 1.5_{\mathrm{C}} / \mathrm{LqhIII}$ lacking $D \mathrm{I}-D \mathrm{II}$ and DII-DIII linkers is composed of three chains which correspond to DI$D I V$. The MODELLER software (ver. 9.22) was used to insert missing residues and sidechains within the polypeptide chains, followed by quick refinement using MD with simulated annealing ${ }^{56}$. Neutral $\mathrm{N}$ - and C-termini were used for the three polypeptide chains in our refined model of $\mathrm{rNa}_{\mathrm{V}} 1.5_{\mathrm{C}}$. N-termini from chains DII and DIII-IV were acetylated, and a neutral amino terminus $\left(-\mathrm{NH}_{2}\right)$ was used for $D I$. Neutral carboxyl groups $(-\mathrm{COOH})$ were used for all C-termini. Disulfide bonds linking residues 327-342, 909-918, and 1730-1744 were included in our model of the channel as they were present in the cryo-EM structure; however, no glycans were added to the protein. Charged N- and C-termini were used for LqhIII and disulfide bonds linking residues $12-65,16-37,23-47$, and $27-49$ were included.

Molecular dynamics simulations. Molecular models of $\mathrm{rNa}_{\mathrm{V}} 1.5_{\mathrm{C}} / \mathrm{LqhIII}$ with and without the toxin were prepared using the input generator, Membrane Builder from CHARMM-GUI ${ }^{57-61}$, from CHARMM-GUI (Jo et al., 2009). The $\mathrm{rNa}_{\mathrm{V}} 1.5_{\mathrm{C}} /$ LqhIII model was embedded in a hydrated DMPC bilayer, with approximately $150 \mathrm{mM} \mathrm{NaCl}$. The protein was translated and rotated for membrane embedding using the PPM server ${ }^{62}$. The lipid bilayer was assembled using the replacement method, and solvent ions were added at random positions using a distance-based algorithm. A periodic rectangular cell with approximate dimensions of $14 \times 14 \times$ $13 \mathrm{~nm}$ was used, which comprised $\sim 240,000$ atoms.

The CHARMM36 all-atom force field ${ }^{63-65}$ was used in conjunction with the TIP3P water model ${ }^{66}$. Non-bonded fixes for backbone carbonyl oxygen atoms with $\mathrm{Na}^{+67}$, and lipid head groups with $\mathrm{Na}^{+68}$ were imposed. Electrostatic interactions were calculated using the particle-mesh Ewald algorithm ${ }^{69,70}$ and chemical bonds were constrained using the LINCS algorithm ${ }^{71}$.

The energy of the system was minimized with protein position restraints on the backbone $\left(4000 \mathrm{~kJ} / \mathrm{mol} / \mathrm{nm}^{2}\right)$ and side chains $\left(2000 \mathrm{~kJ} / \mathrm{mol} / \mathrm{nm}^{2}\right)$, as well as lipid position and dihedral restraints $\left(1000 \mathrm{~kJ} / \mathrm{mol} / \mathrm{nm}^{2}\right)$ using 5000 steps of steepest descent. The simulation systems were pre-equilibrated using multistep isothermal-isovolumetric (NVT) and isothermal-isobaric (NPT) conditions for a total of $10.35 \mathrm{~ns}$ (see Table "MDS1 for parameters"). Unrestrained "production" simulations of approximately $300 \mathrm{~ns}$ were then generated with a $2 \mathrm{fs}$ time integration step. The first $100 \mathrm{~ns}$ of all production simulations were considered part of equilibration based on RMSD analyses of Ca atoms (Supplementary Fig. 6) and were excluded from subsequent data analysis. Thirty independent replicas (10 of them $400 \mathrm{~ns}$-long and 20 of them $300 \mathrm{~ns}$ long) were generated for each system using random starting velocities, yielding a total simulation time of approximately $10.3 \mu$ s per system, of which $7.3 \mu$ s were used for analysis. The simulations were carried out using GROMACS version 2019.3 (http://www.gromacs.org).

Molecular dynamics simulation analysis. In each snapshot of our simulations, atomic positions were translated and rotated by aligning the $\mathrm{Ca}$ atoms from pore transmembrane helices ( $\mathrm{S} 5$ and $\mathrm{S} 6$ from all domains) of $\mathrm{rNa}_{\mathrm{V}} 1.5_{\mathrm{C}}$ to the initial structure produced by CHARMM-GUI. The positions of all atoms were then centered in the xy-plane by the center of mass $(\mathrm{CoM})$ of pore transmembrane helices and the $z$-axis by the CoM of Ca atoms from the DEKA motif in the SF. After performing the spatial transformations, the $z$-axis of the simulation box was used as the pore axis of $\mathrm{Na}_{\mathrm{V}} 1.5$ and the transformed positions were used for subsequent analyses.

The axial distribution of water was computed by counting the number of water O-atoms within a cylindrical radius $8.5 \AA$ centered on the pore axis. The probability distribution of water was calculated for each replica by counting the number of water molecules in uniform cylindrical slices along the pore-axis and normalizing the counts by the slice with the highest number of water molecules (solvent slice). The average and SEM of the probability distribution was computed across replicas.

Pore hydration analysis indicated a dehydrated region located at the ICAG $(-2.8 \mathrm{~nm}<z<-1.5 \mathrm{~nm})$. The number of water molecules in the gate was counted for each frame and normalized by the total number of frames to obtain the probability distribution. The average and s.e.m. were computed across replicas.

To measure the size of the intracellular activation gate, residues at the ends of the S6 helices were selected by using a similar residue selection as Lenaeus et al. in their study of open- and closed-state $\mathrm{Na}_{\mathrm{V}} \mathrm{Ab}$ structures ${ }^{72}$. Because $\mathrm{rNa}_{\mathrm{V}} 1.5_{\mathrm{C}}$ has $94 \%$ sequence similarity with $\mathrm{hNa} \mathrm{V}_{\mathrm{V}} 1.5$ (gap open penalty of 12 , gap extension penalty of 4), a previously published multiple sequence alignment of $\mathrm{Na}_{\mathrm{V}} \mathrm{Ab}$ to $\mathrm{hNa}_{\mathrm{V}} 1.5 \mathrm{~S} 6$ helices by ${ }^{73}$ was used to determine the equivalent residues in $\mathrm{rNa}_{\mathrm{V}} 1.5$. As a result, the following residue number selections were used in $\mathrm{rNa}_{\mathrm{v}} 1.5$ : DI: 410-413, DII: 939-942, DIII: 1469-1472, and DIV: 1771-1774. The CoM of Ca-atoms from each selection was projected onto the xy-plane and the distances between opposing S6 tails were measured $\left(d_{1}\right.$ : DI-DIII and $d_{2}$ : DII-DIV).

Analyses were performed using MDTraj ${ }^{74}$ and molecular visualizations were rendered using Visual Molecular Dynamics ${ }^{75}$.

Reporting summary. Further information on research design is available in the Nature Research Reporting Summary linked to this article.

\section{Data availability}

Data supporting the findings of this paper are available from the corresponding authors upon reasonable request. A reporting summary for this Article is available as a Supplementary Information file. Structural Data are available from the Protein Data Bank under EMDB entry ID EMD-2262 and PDB entry ID 7K18. Source data are provided with this paper.

Received: 3 August 2020; Accepted: 10 November 2020; Published online: 04 January 2021

\section{References}

1. Hille, B. Ionic Channels of Excitable Membranes, 3rd Ed. (Sinauer Associates Inc., 2001).

2. Hodgkin, A. L. \& Huxley, A. F. A quantitative description of membrane current and its application to conduction and excitation in nerve. J. Physiol. 117, 500-544 (1952).

3. Catterall, W. A. Neurotoxins that act on voltage-sensitive sodium channels in excitable membranes. Annu. Rev. Pharmacol. Toxicol. 20, 15-43 (1980).

4. Catterall, W. A. et al. Voltage-gated ion channels and gating modifier toxins. Toxicon 49, 124-141 (2007).

5. Lazdunski, M. et al. Polypeptide toxins as tools to study voltage-sensitive $\mathrm{Na}+$ channels. Ann NY Acad Sci 479, 204-220 (1986).

6. Zlotkin, E., Miranda, F. \& Lissitzky, S. Proteins in scorpion venoms toxic to mammals and insects. Toxicon 10, 207-209 (1972).

7. Rochat, H., Bernard, P. \& Couraud, F. Scorpion toxins: chemistry and mode of action. Adv Cytopharmacol 3, 325-334 (1979).

8. Romey, G., Abita, J. P., Chicheportiche, R., Rochat, H. \& Lazdunski, M Scorpion neurotoxin. Mode of action on neuromuscular junctions and synaptosomes. Biochim Biophys Acta 448, 607-619 (1976).

9. Rogers, J. C., Qu, Y., Tanada, T. N., Scheuer, T. \& Catterall, W. A. Molecular determinants of high affinity binding of alpha-scorpion toxin and sea anemone toxin in the S3-S4 extracellular loop in domain IV of the $\mathrm{Na}+$ channel alpha subunit. J. Biol. Chem. 271, 15950-15962 (1996).

10. Sheets, M. F., Kyle, J. W., Kallen, R. G. \& Hanck, D. A. The Na channel voltage sensor associated with inactivation is localized to the external charged residues of domain IV, S4. Biophys. J. 77, 747-757 (1999).

11. Wang, J. et al. Mapping the receptor site for alpha-scorpion toxins on a $\mathrm{Na}+$ channel voltage sensor. Proc Natl Acad Sci USA 108, 15426-15431 (2011).

12. Chanda, B. \& Bezanilla, F. Tracking voltage-dependent conformational changes in skeletal muscle sodium channel during activation. J. Gen. Physiol 120, 629-645 (2002).

13. Capes, D. L., Goldschen-Ohm, M. P., Arcisio-Miranda, M., Bezanilla, F. \& Chanda, B. Domain IV voltage-sensor movement is both sufficient and rate limiting for fast inactivation in sodium channels. J. Gen. Physiol 142, 101-112 (2013).

14. Catterall, W. A., Wisedchaisri, G. \& Zheng, N. The chemical basis for electrical signaling. Nature Chem. Biol. 13, 455-463 (2017)

15. Ahern, C. A., Payandeh, J., Bosmans, F. \& Chanda, B. The hitchhiker's guide to the voltage-gated sodium channel galaxy. J. Gen. Physiol. 147, 1-24 (2016).

16. Payandeh, J., Scheuer, T., Zheng, N. \& Catterall, W. A. The crystal structure of a voltage-gated sodium channel. Nature 475, 353-358 (2011).

17. Zhang, X. et al. Crystal structure of an orthologue of the NaChBac voltagegated sodium channel. Nature 486, 130-134 (2012). [pii].

18. Shaya, D. et al. Structure of a prokaryotic sodium channel pore reveals essential gating elements and an outer ion binding site common to eukaryotic channels. J. Mol. Biol. 426, 467-483 (2014).

19. Pan, X. et al. Structure of the human voltage-gated sodium channel NaV1.4 in complex with beta1. Science 362, eaau2486 (2018). pii.

20. Shen, H., Liu, D., Wu, K., Lei, J. \& Yan, N. Structures of human NaV1.7 channel in complex with auxiliary subunits and animal toxins. Science 363, 1303-1308 (2019) 
21. Jiang, D. et al. Structure of the cardiac sodium channel. Cell 180, 122-134 (2020).

22. Gamal El-Din, T. M., Lenaeus, M. J. \& Catterall, W. A. Structural and functional analysis of sodium channels viewed from an evolutionary perspective. Handb Exp Pharmacol 246, 53-72 (2018).

23. Cestele, S. et al. Structure and function of the voltage sensor of sodium channels probed by a beta-scorpion toxin. J. Biol. Chem. 281, 21332-21344 (2006). M603814200.

24. Catterall, W. A. Binding of scorpion toxin to receptor sites associated with sodium channels in frog muscle. Correlation of voltage-dependent binding with activation. J. Gen. Physiol 74, 375-391 (1979).

25. Catterall, W. A. Membrane potential-dependent binding of scorpion toxin to the action potential $\mathrm{Na}+$ ionophore. Studies with a toxin derivative prepared by lactoperoxidase-catalyzed iodination. J. Biol. Chem. 252, 8660-8668 (1977).

26. Clairfeuille, T. et al. Structural basis of alpha-scorpion toxin action on Nav channels. Science, 10.1126/science.aav8573 (2019).

27. Gilles, N. et al. A scorpion alpha-like toxin that is active on insects and mammals reveals an unexpected specificity and distribution of sodium channel subtypes in rat brain neurons. J. Neurosci. 19, 8730-8739 (1999).

28. Chen, H. \& Heinemann, S. H. Interaction of scorpion alpha-toxins with cardiac sodium channels: binding properties and enhancement of slow inactivation. J. Gen. Physiol 117, 505-518 (2001).

29. Krimm, I. et al. NMR structures and activity of a novel alpha-like toxin from the scorpion Leiurus quinquestriatus hebraeus. J Mol Biol 285, 1749-1763 (1999).

30. Rahim, A., Saha, P., Jha, K. K., Sukumar, N. \& Sarma, B. K. Reciprocal carbonyl-carbonyl interactions in small molecules and proteins. Nat Commun 8, 78 (2017)

31. Wisedchaisri, G. et al. Resting-state structure and gating mechanism of a voltage-gated sodium channel. Cell 178, 993-1003 (2019).

32. Lenaeus, M. J. et al. Structures of closed and open states of a voltage-gated sodium channel. Proc Natl Acad Sci USA 114, E3051-E3060 (2017).

33. Chakrabarti, N. et al. Catalysis of $\mathrm{Na}+$ permeation in the bacterial sodium channel NaVAb. Proc Natl Acad Sci USA 110, 11331-11336 (2013).

34. Jensen, M. O. et al. Principles of conduction and hydrophobic gating in $\mathrm{K}+$ channels. Proc Natl Acad Sci USA 107, 5833-5838 (2010).

35. Neale, C., Chakrabarti, N., Pomorski, P., Pai, E. F. \& Pomes, R. Hydrophobic gating of ion permeation in magnesium channel CorA. PLoS Comput Biol 11, e1004303 (2015).

36. Aryal, P., Sansom, M. S. \& Tucker, S. J. Hydrophobic gating in ion channels. J Mol Biol 427, 121-130 (2015).

37. Zhu, F. \& Hummer, G. Drying transition in the hydrophobic gate of the GLIC channel blocks ion conduction. Biophys J 103, 219-227 (2012).

38. Catterall, W. A. Molecular properties of voltage-sensitive sodium channels. Annu.Rev.Biochem. 55, 953-985 (1986).

39. Yarov-Yarovoy, V. et al. Structural basis for gating charge movement in the voltage sensor of a sodium channel. Proc. Natl. Acad. Sci. USA 109, E93-E102 (2012). 1118434109.

40. Pan, X. et al. Molecular basis for pore blockade of human $\mathrm{Na}+$ channel NaV1.2 by the mu-conotoxin KIIIA. Science 363, 1309-1313 (2019).

41. Catterall, W. A. \& Beress, L. Sea anemone toxin and scorpion toxin share a common receptor site associated with the action potential sodium ionophore. J.Biol.Chem. 253, 7393-7396 (1978).

42. Benzinger, G. R., Kyle, J. W., Blumenthal, K. M. \& Hanck, D. A. A specific interaction between the cardiac sodium channel and site- 3 toxin anthopleurin B. J.Biol.Chem. 273, 80-84 (1998).

43. Renaud, J. F., Fosset, M., Schweitz, H. \& Lazdunski, M. The interaction of polypeptide neurotoxins with tetrodotoxin-resistant $\mathrm{Na}+$ channels in mammalian cardiac cells. Correlation with inotropic and arrhythmic effects. Eur J Pharmacol 120, 161-170 (1986).

44. Couraud, F., Jover, E., Dubois, J. M. \& Rochat, H. Two types of scorpion toxin receptor sites, one related to the activation, the other to the activation of the action potential sodium channel. Toxicon 20, 9-16 (1982).

45. Cestèle, S. et al. Voltage sensor-trapping: Enhanced activation of sodium channels by beta-scorpion toxin bound to the S3-S4 loop in domain II. Neuron 21, 919-931 (1998).

46. Zhang, J. Z. et al. Structure-function map of the receptor site for beta-scorpion toxins in domain II of voltage-gated sodium channels. J Biol Chem 286, 33641-33651 (2011). M111.282509.

47. Zhang, J. Z. et al. Mapping the interaction site for a beta-scorpion toxin in the pore module of domain III of voltage-gated $\mathrm{Na}+$ channels. J Biol Chem 287, 30719-30728 (2012). M112.370742.

48. Saez, N. J. et al. Spider-venom peptides as therapeutics. Toxins (Basel) 2, 2851-2871 (2010).

49. Suloway, C. et al. Automated molecular microscopy: the new leginon system. J. Struc. Biol. 151, 41-60 (2005).

50. Zheng, S. Q. et al. MotionCor2: anisotropic correction of beam-induced motion for improved cryo-electron microscopy. Nat. Methods 14, 331-332 (2017).

51. Scheres, S. H. W. RELION: Implementation of a Bayesian approach to cryoEM structure determination. J. Stru. Biol. 180, 519-530 (2012).
52. Pettersen, E. F. UCSF Chimera? A visualization system for exploratory research and analysis. J. Comput. Chem. 25, 1605-1612 (2004).

53. Zhang, K. Gctf: Real-time CTF determination and correction. J. Struc. Biol. 193, 1-12 (2016).

54. Emsley, P., Lohkamp, B., Scott, W. G. \& Cowtan, K. Features and development of coot. Acta Crystallogr. D Biol. Crystallogr. 66, 486-501 (2010).

55. Adams, P. D. et al. PHENIX: a comprehensive Python-based system for macromolecular structure solution. Acta Crystallogr. D Biol. Crystallogr. 66, 213-221 (2010).

56. Fiser, A. \& Sali, A. Modeller: generation and refinement of homology-based protein structure models. Meth. Enzymol. 374, 461-491 (2003).

57. Jo, S., Kim, T. \& Im, W. Automated builder and database of protein/membrane complexes for molecular dynamics simulations. PLoS ONE 2, e880 (2007).

58. Jo, S., Lim, J. B., Klauda, J. B. \& Im, W. CHARMM-GUI membrane builder for mixed bilayers and its application to yeast membranes. Biophys. J. 97, 50-58 (2009).

59. Lee, J. et al. CHARMM-GUI input generator for NAMD, GROMACS, AMBER, OpenMM, and CHARMM/OpenMM simulations using the CHARMM36 additive force field. J. Chem. Theory Comput. 12, 405-413 (2015).

60. Lee, J. et al. CHARMM-GUI for complex biological membrane simulations with glycolipids and lipoglycans. J. Chem. Theory Comput. 15, 775-786 (2018).

61. Wu, E. L. et al. CHARMM-GUI toward realistic biological membrane simulations. J. Comput. Chem. 35, 1997-2004 (2014).

62. Lomize, M. A. et al. OPM database and PPM web server: resources for positioning of proteins in membranes. Nucleic Acids Res. 40, E370-D376 (2012)

63. Best, R. B. et al. Optimization of the additive CHARMM all-atom protein force field targeting improved sampling of the backbone $\phi, \psi$ and Side-Chain $\chi$ and $\chi$ dihedral angles. J. Chem. Theory Comput. 8, 3257-3273 (2012).

64. Klauda, J. B. et al. Update of the CHARMM all-atom additive force field for lipids: validation on six lipid types. J. Phys. Chem. B 114, 7830-7843 (2010).

65. MacKerell, J. B. et al. All-atom empirical potential for molecular modeling and dynamics studies of proteins. J. Phys. Chem. B 102, 3586-3616 (1998).

66. Jorgensen, W. L. et al. Comparison of simple potential functions for simulating water. J. Chem. Phys. 79, 926-935 (1983).

67. Noskov, S. Y. \& Roux, B. Control of ion selectivity in LeuT: two Na+ binding sites with two different mechanisms. J. Mol. Biol. 377, 804-818 (2008)

68. Venable, R. M., Luo, Y., Gawrisch, K., Roux, B. \& Pastor, R. W. Simulations of anionic lipid membranes: development of interaction-specific ion parameters and validation using NMR data. J. Chem. Phys. B 117, 10183-10192 (2013).

69. Darden, T., York, D. \& Pedersen, L. Particle mesh Ewald: an $N \log (N)$ method for Ewald sums in large systems. J. Chem. Phys. 98, 10089-10092 (1993).

70. Essmann, T. et al. A smooth particle mesh Ewald method. J. Chem. Phys. 103, 8577-8593 (1995).

71. Hess, B. P-LINCS: a parallel linear constraint solver for molecular simulation. J. Chem. Theory Comput. 4, 116-122 (2007)

72. Lenaeus, M. J. et al. Structures of closed and open states of a voltage-gated sodium channel. Proc. Natl Acad. Sci. USA 114, E3051-E3060 (2017).

73. Boiteux, C., Vorobyov, I., French, R. J., French, C., Yarov-Yarovoy, V. \& Allen, $\mathrm{T}$. W. Local anesthetic and antiepileptic drug access and binding to a bacterial voltage-gated sodium channel. Proc. Natl Acad. Sci. USA 111, 13057-13062 (2014).

74. McGibbon, R. T. et al. MDTraj: a modern open library for the analysis of molecular dynamics trajectories. Biophys. J. 109, 1528-1532 (2015).

75. Humphrey, W., Dalke, A. \& Schulten, K. VMD: Visual molecular dynamics. J. Mol. Graph. 14, 33-38 (1996).

\section{Acknowledgements}

We thank J.D. Quispe and Quinton Beedle of the University of Washington Cryo-EM Facility for advice and technical assistance during data collection and $\mathrm{Dr}$. Jin $\mathrm{Li}$, Department of Pharmacology, University of Washington, for technical and editorial support. This research was supported by National Institutes of Health Research Grant R01 HL112808 (W.A.C. and N.Z.) and by the Howard Hughes Medical Institute (N.Z.), and by Canadian Institutes of Health Research grant MOP130461 (R.P.). Molecular simulations were enabled by supercomputing resources and support provided by WestGrid (www.westgrid.ca), SciNet (www.scinet.ca), and Compute Canada (www. computecanada.ca).

\section{Author contributions}

D.J., L.T., T. M.G., R.B., R.P., N.Z., and W.A.C. designed the experiments, D.J. and L.T carried out the cryo-EM experiments; T.M.G. carried out the electrophysiology experiments; R.B. and R.P. carried out the molecular dynamics simulations and analyses, and all authors contributed to writing and revising the paper.

\section{Competing interests}

The authors declare no competing interests. 


\section{Additional information}

Supplementary information is available for this paper at https://doi.org/10.1038/s41467020-20078-3.

Correspondence and requests for materials should be addressed to N.Z. or W.A.C.

Peer review information Nature Communications thanks Alexander Sobolevsky and other, anonymous, reviewers for their contributions to the peer review of this work.

Reprints and permission information is available at http://www.nature.com/reprints

Publisher's note Springer Nature remains neutral with regard to jurisdictional claims in published maps and institutional affiliations. (c) (i) Open Access This article is licensed under a Creative Commons Attribution 4.0 International License, which permits use, sharing, adaptation, distribution and reproduction in any medium or format, as long as you give appropriate credit to the original author(s) and the source, provide a link to the Creative Commons license, and indicate if changes were made. The images or other third party material in this article are included in the article's Creative Commons license, unless indicated otherwise in a credit line to the material. If material is not included in the article's Creative Commons license and your intended use is not permitted by statutory regulation or exceeds the permitted use, you will need to obtain permission directly from the copyright holder. To view a copy of this license, visit http://creativecommons.org/ licenses/by/4.0/.

(C) The Author(s) 2021 